\title{
Fungal and Mycotoxin Contamination of Nigerian Foods and Feeds
}

\author{
Olusegun Atanda, Hussaini Anthony Makun, \\ Isaac M. Ogara, Mojisola Edema, Kingsley O. Idahor, \\ Margaret E. Eshiett and Bosede F. Oluwabamiwo
}

Additional information is available at the end of the chapter

http://dx.doi.org/10.5772/55664

\section{Introduction}

Fungi are ubiquitous plant pathogens that are major spoilage agents of foods and feedstuffs. The infection of plants by various fungi not only results in reduction in crop yield and quality with significant economic losses but also contamination of grains with poisonous fungal secondary metabolites called mycotoxins. The ingestion of such mycotoxincontaminated grains by animals and human beings has enormous public health significance, because these toxins are capable of causing diseases in man and animals (Bhat and Vasanthi 2003). Although the involvement of fungi and their toxins in causing diseases to man and animals dates back to the period when the Dead Sea Scrolls were written (Richard, 2007) it seems the evidence of their historic occurrence and impact were not obvious until the Middle Ages, when ergot alkaloids poisoning outbreaks in Europe were responsible for the death of thousands of people. Subsequently, between 1940s and 1950s a lethal human disease caused by Fusarium toxins and referred to as 'Alimentary Toxic Aleukia' was reported in Russia (Smith and Moss, 1985). Similarly in 1938 in Japan, Penicillium species were responsible for the colouring of rice that erratically led to the fatal human cardiac syndrome called 'yellow rice disease' (Uraguchi and Yamazaki, 1978). The livestock industry was also affected as seen by the devastation of the New Zeland sheep industry by facial eczema a fungal infection caused by Pithomyces chartarum in 1822. Other deadly animal syndromes arising from fungal infections and termed differently as equine leukoencephalomalacia (1930 to 1970 in USA), stachybotryotoxicosis (1930 in USSR), red mould diseases (1945-1947 in Japan), and red clover disease, vulvovaginitis and mouldy corn toxicosis (1920 to 1950 in USA) plagued the world (Gbodi and Nwude, 1988). In spite of these episodes little attention was paid to fungal diseases. However, in 1960, when the Turkey X disease killed thousands of poultry animals in Britain (Blount, 1965); the world 
became fully aware of the potential hazards of mycotoxins and responded to the disaster by a systematic and multidisciplinary approach which led to the discovery of aflatoxins as deadly contaminant of groundnuts.

Following the outbreak of aflatoxicosis and the enormous economic loss in the poultry industry of Britain in 1961, the Federal government of Nigeria, in order to protect her export trade initiated screening studies to determine the extent of aflatoxin contamination of groundnut and groundnut products ( Blount, 1961; Darling, 1963, Haliday, 1965, 1966 and Halliday and Kazaure, 1967). McDonald and Harkness (1965) found aflatoxins in groundnut samples from Zaria, Kano and Mokwa, in Northern Nigeria. Bassir and Adekunle (1968) isolated two metabolites of Aspergillus flavus from palm wine which were characterized and named palmotoxin Bo and Go. The toxicity of palmotoxin Bo was comparable to that of aflatoxin $B_{1}$ in embryonated eggs (Bassir and Adekunle, 1969). Bassir (1969) also isolated aflatoxin $B_{1}$ from various mouldy food materials offered for sale in Ibadan markets. Since then, toxigenic fungi and mycotoxins have been found in various foods and feedstuffs in many regions of Nigeria (Tables 1 \& 2) thus mycotoxigenic fungi belonging to not less than forty five fungal genera and about twenty different mycotoxins have been detected in Nigerian foods and foodstuffs. Two cases of animal mycotoxicoses (Ikwuegbu , 1984 and Ocholi et al. 1992) and two suspected cases of human mycotoxicosis were also reported in the country. The animal mycotoxicosis involved pecking ducklings and horses while the human mycotoxicosis involved primary school pupils that consumed groundnut cake ("kulikuli") in Ibadan ( Akano \& Atanda, 1990).

The presence of mycotoxins in our food systems and tissues has enormous public health significance because these toxins are nephrotoxic, immunotoxic, teratogenic and mutagenic. They are also capable of causing acute and chronic effects in man and animals ranging from death to disorder of central nervous, cardiovascular, pulmonary systems and intestinal tract (Bhat and Vasanthi, 2003). Of greatest concern is the relevance of these toxins in human hepatoma and oesophageal cancer, increased susceptibility to diseases especially in children and childhood pre-five mortality and reduced life expectancy (Beardall and Miller, 1994, Miller, 1996 and, Marasas, 2001).

Furthermore, Nigeria has experienced high recorded aflatoxin exposure levels in humans and has also reported the highest estimated number of cases of hepatocellular carcinoma (HCC-liver cancer) attributable to aflatoxins (Liu and $\mathrm{Wu}, 2010)$ in the whole world.

Due to the insidious nature of mycotoxin production and the resulting disease states which made diagnosis of mycotoxicosis difficult; many cases of both human and animal mycotoxicoses have often not been reported in Nigeria. This suggests that little has been done on mycotoxicosis in Nigeria and there is paucity of information on mycotoxins in the country.

In view of the negative public health and economic impacts of mycotoxins this chapter, written by members of the Mycotoxicology Society of Nigeria, the first of such society in Africa will examine the factors that influence the development of mycotoxin producing fungi and mycotoxin production and provide a current overview of the natural incidence of 
these toxins in different raw and processed food commodities that serve as principal sources of the toxins. It will also examine the human health hazards of the toxins with particular reference to the Nigerian situation as well as review the control and regulatory strategies possible within the country's technological capacity among others.

\section{Factors affecting the incidence of mycotoxigenic fungi and mycotoxins in Nigeria}

Various classifications are used in categorizing the factors that affect the incidence of mycotoxigenic fungi and mycotoxins in the food chain. Some classifications categorize these factors as extrinsic and intrinsic, some as physical, chemical and biological factors while others classify them as ecological, environmental and storage factors (D'Mello and MacDonald, 1997; Zain, 2011). Irrespective of the form of classification, Lacey (1986) identified the key elements involved in stating that the type and amount of mycotoxin produced is always determined by the fungi, substrate and environmental factors. In Nigeria, without necessarily sticking with any of the classification systems above, we can group the factors into 15 types as outlined below.

\subsection{Climatic conditions}

Probably the two most important environmental components favouring mold growth and mycotoxin production are hot and humid conditions. Mycotoxins occur more frequently in areas with a hot and humid climate, favourable for the growth of moulds. Although they can also be found in temperate zones, tropical climates such as those existing in Nigeria have been found to be quite conducive for mould growth and mycotoxin production. Mycotoxigenic fungi are most abundant in the tropics and as such, are major food spoilage agents in these warmer climates (Mclean and Berjak, 1987). Although the optimum temperature and moisture content for growth and toxin production for the various toxigenic fungi vary, many of them achieve best growth and toxin synthesis between $24^{\circ} \mathrm{C}$ and $28^{\circ} \mathrm{C}$ and seed moisture content of at least 17.5\% (Trenk and Hartman, 1970; Ominski et al., 1994). These conditions approximate the ambient climatic conditions in most parts of Africa and hence also account for the high prevalence of the toxins on the continent. Drought conditions actually constitute stress factors to plants rendering them vulnerable to mould infection with ensuing increase in toxin production. An indelible sign that droughts prop up toxin contamination is the fact that these conditions preceded the fatal outbreak of acute human aflatoxicosis that occurred in Kenya in 2004 (CDC, 2004). Edema and Adebanjo (2000) and Makun et al (2009a, b) recorded higher mycotoxigenic fungal contamination during the rainy season than in the dry harmattan season among produce in Nigeria.

\subsection{Availability of nutrients and conditions for mould growth}

The fact that a strain of mould has the genetic potential to produce a particular mycotoxin is not enough for it to do so. There must be enough nutrients to encourage mould growth and the level of mycotoxin production would in part be influenced by the nutrients available to 
the mould. Typically, moulds require a source of energy in the form of carbohydrates or vegetable oils in addition to a source of nitrogen either organic or inorganic, trace elements and available moisture for growth and toxin production. Substrate may also play a role in selecting for or against toxin producing strains of a given species, e.g., there is a high proportion of toxin-producing strains of $A$. flavus isolated from peanuts and cottonseed than from rice or sorghum. It has also been found that strains of ochratoxin and citrininproducing $P$. viridicatum isolated from meat were more unstable than those isolated from grain and rapidly lost toxin-producing ability. Field fungi like Fusarium and Alternaria contaminate grains before or during harvest. The storage fungi (e.g. Penicillium and Aspergillus) are capable of growing at lower water content than the field fungi and they tend to contaminate the grains in silos and other storage places. It is known that aflatoxin production is favoured by prolonged end of season drought and associated elevated temperatures (Rachaputi et al., 2002). Moulds can grow and produce mycotoxins under a wide temperature range with optima generally between 20 to $30^{\circ} \mathrm{C}$. However, temperatures optimum for toxin production need not correspond to those optimum for growth: Fusarium tricinctum grows well at $25^{\circ} \mathrm{C}$ but produces $\mathrm{T}-2$ toxin best near freezing temperatures. Penicillium martensii produces penicillic acid rapidly at $20-30^{\circ} \mathrm{C}$, but considerably more toxin eventually accumulates between 4 to $10^{\circ} \mathrm{C}$.

\subsection{Farming systems and agricultural techniques}

A number of farming techniques have been shown in various reports as stimulating mould growth in agricultural produce. For example produce harvested from land on which groundnut has been planted the previous year were infested more by Aspergillus flavus and contained more aflatoxin than crops grown on land previously planted with rye, oats, melon or potatoes indicating that crop rotation influences mycotoxigenic mould growth. Likewise, previously fungicide-treated soil has been shown to reduce incidence of A. flavus in groundnuts to very low levels.

\subsection{Soil types and soil conditions}

Soil is a natural factor that exerts a powerful influence on the incidence of fungi. Crops grown in different soil types may have significantly different levels of mycotoxin contamination. For example, peanuts grown in light sandy soils support rapid growth of the fungi, particularly under dry conditions, while heavier soils result in less contamination of peanuts due to their high water holding capacity which helps the plant to prevent drought stress (Codex Alimentarius Commission, 2004).

\subsection{Pre-harvest conditions}

Genotypes, drought, soil type, plant density, fertilization level, and insect activities are important components in determining the likelihood of pre-harvest contamination (Cole et al., 1995). However, the most important factor appears to be high night time temperatures, which 
favour fungal growth and toxin production at a time when the plant is deprived of its usual energy source and thus least able to resist fungal attack (Abbas et al., 2002, 2007; Payne, 1992).

\subsection{Time of harvesting}

Harvest is the first stage in the production chain where moisture content becomes the most important parameter in terms of the management and protection of the crop. It also marks a shift from problems caused by plant pathogenic fungi, like Fusarium, to problems caused by storage fungi, like Penicillium verrucosum. Ideally, grains will always be harvested after a spell of dry weather when it is at a 'safe' moisture content, so that immediate drying is not necessary. However, this is not always possible hence inappropriate harvest time is a risk factor in Nigeria. Another important control measure at harvest will be visual examination of the grain for symptoms of disease, and the segregation of diseased batches from healthy grain. Early harvesting reduces fungal infection of crops in the field and consequent contamination of harvested produce. Even though majority of farmers in Africa are well aware of the need for early harvesting, lack of storage space, unpredictable weather, labour constraint, need for cash, threat of thieves, rodents and other animals compel farmers to harvest at inappropriate time (Bankole and Adebanjo, 2003). Kaaya et al. (2006) observed that aflatoxin levels increased by about 4 times by the third week and more than 7 times when maize harvest was delayed for 4 weeks. However, If products are harvested early, they have to be dried to safe levels to stop fungal growth. Rachaputi et al. (2002) reported lower aflatoxin levels and higher gross returns of $27 \%$ resulting from early harvesting and threshing of groundnuts.

\subsection{Pest infestation}

Insects are the chief causes of deterioration and loss of grains and seeds. Their invasion of cereals decreases the quality, grade and market value of these agricultural products which in most instances are rendered unsafe for human and animal consumption. Pest infestation is largely due to improper post-harvest and storage conditions and the level of insect damage influences the extent of mycotoxin contamination. Avantaggio et al. (2002) found that insect damage of maize is good predictor of Fusarium mycotoxin contamination. Insects carry spores of mycotoxin-producing fungi from plant surfaces to the interior of the stalk or kernels or create infectious wounds through their feeding habits (Munkvold, 2003).

\subsection{Post-harvest handling}

The post-harvest stages are those stages following harvest and leading up to primary processing such as milling. This will typically involve drying (if required), storage and transportation steps. Post-harvest movement of food/feed commodities can be complex, passing as it may between a number of intermediaries such as traders and intermediate processors, who may be situated at different geographical locations. In the simplest case, produce may remain on-farm in store or buffer storage for short periods of time before being passed directly onto the processor. In more complex cases it may pass through the 
hands of merchants or third party drying facilities (if harvested wet e.g. grains) and held in storage for periods of time before finally arriving at the processors. At all times the produce can become susceptible to fungal contamination and mycotoxin production if the storage conditions are not strictly controlled.

\subsection{Drying conditions and duration}

Rapid drying of agricultural products to low moisture level is critical as it creates less favourable conditions for fungal growth, proliferation, and insect infestation. It helps keep products longer (Lanyasunya et al., 2005). Ayodele and Edema (2010, In Press) evaluated the Critical Control Points (CCP) in the production of dried yam chips with a view to reducing mycotoxin contamination and identified the drying stage as a CCP. Aflatoxin contamination can increase 10 fold in a 3-d period, when field harvested maize is stored with high moisture content (Hell et al., 2008). The general recommendation is that harvested commodities should be dried as quickly as possible to safe moisture levels of $10-13 \%$. Achieving this through simple sun-drying under the high humidity conditions of many parts of Africa, such as the humid southern Nigeria is very difficult. Even, when drying is done in the dry season, it is not completed before loading grains into stores as observed by Mestre et al. (2004) and products can be easily contaminated with aflatoxins. During storage, transportation and marketing, low moisture levels should be maintained by avoiding leaking roofs and condensation arising from inadequate ventilation.

\subsection{Storage factors}

Mycotoxin contamination of foods or feeds may result from inadequate storage and/or handling of harvested products. To preserve quality in storage, it is necessary to prevent biological activity through adequate drying to less than $10 \%$ moisture, elimination of insect activity that can increase moisture content through condensation of moisture resulting from respiration, low temperatures, and inert atmospheres (Lanyasunya et al., 2005; Turner et al., 2005). Several field and storage fungi have been reported in Nigeria (Tables $1 \& 2$ ) and the post-harvest contamination is normally characterized by the activities of the 'storage' fungi, typically Aspergillus and Penicillium species that are able to grow in relatively dry conditions.

\subsection{Sanitation}

Basic sanitation measures such as removal and destruction of debris from previous harvest would help in minimizing infection and infestation of produce in the field. Sorting out physically damaged and infected grains (known from colorations, odd shapes and size) from the intact commodity can result in $40-80 \%$ reduction in aflatoxins levels

\subsection{Traditional processing methods}

A study conducted in Benin by Fandohan et al. (2005) to determine the fate of aflatoxins and fumonisins through traditional processing of naturally-contaminated maize and maizebased foods, demonstrated that sorting, winnowing, washing, crushing combined with de- 
hulling of maize grains were effective in achieving significant mycotoxins removal. Similar results have been reported by Park (2002) and Lopez-Garcia and Park (1998). This approach is based on separation of contaminated grains from the bulk grains and depends on heavy contamination of only a small fraction of the seeds, so that removing those leaves a much.

Wet and dry milling processes as well as heat in the cooking process have been shown to reduce mycotoxin production in foods. Heating and roasting can significantly decrease aflatoxin content in corn. Grain cleaning and further processing in mills can divert mycotoxins to various mill streams, and further processing such as baking may reduce mycotoxin levels. A review of several studies, however, suggested that processing and pasteurization of milk do not completely destroy mycotoxins (Manorama and Singh, 1995).

\subsection{Presence of previous contaminants}

The presence of other microorganisms either bacteria or fungi may alter elaboration of mycotoxins on food materials. When A. parasiticus was grown in the presence of some bacteria; Streptococcus lactis and Lactobacillius casei, aflatoxin production was reduced (Ominski et al., 1994). Meanwhile, fungal metabolites such as rubratoxins from Penicillium purpurogenum; cerulenin from Ephalosporium caerulens and Acrocylindrium oryzae enhance aflatoxin production even though they repress growth of aflatoxin-producing fungi (Smith and Moss, 1985). This type of positive interaction between fungi in the same food matrix with regards to aflatoxin synthesis coupled with multi-occurrence of mycotoxins from the different fungi could have additive or synergistic effect on the health of the host (Speijer and Speijer, 2004) and worsen the aflatoxin plight in Nigeria because such simultaneous co-occurrence of fungi and mycotoxins in African agricultural commodities is a very common phenomenon as indicated by many workers such as Makun et al. (2007), Makun et al. (2009) and Njobeh et al. (2009).

\subsection{Substrate types and properties}

Certain Agricultural produce have been observed to permit the growth of some moulds over others. For example, maize allows the growth of aflatoxins and fumonisins producing moulds above others, while groundnuts have been found to be excellent substrate for aflatoxin contamination (Bankole and Adebanjo, 2003). Other food products for which mycotoxin contamination has been reported in Nigeria are dried yam chips, tiger nut, melon seeds and stored herbal plants. Cereal grains, peanuts, cottonseed and some forages appear to be commongly contaminated with foods and feed substances that may be contaminated with mycotoxins. Similarly, intake of Fusarium toxins, such as trichothecenes and fumonisins, is almost solely due to consumption of cereals).

\subsection{Lack of awareness}

Lack of awareness of the dangers posed by mycotoxin contamination of produce is a major factor responsible for its high incidence in Nigeria. Majority of farmers produce and food handlers and/or processors are illiterate with virtually no knowledge of the implications of toxigenic mould growth. The Mycotoxicology Society of Nigeria has done a lot to reverse 
this trend. The stake holders believe that the powdery substance can be easily dusted off or rinsed with water before the food material is eaten or processed for consumption with no associated risks. The contaminated, mould infested produce are proudly displayed on market stalls (Figs 1 \& 2) for sale.

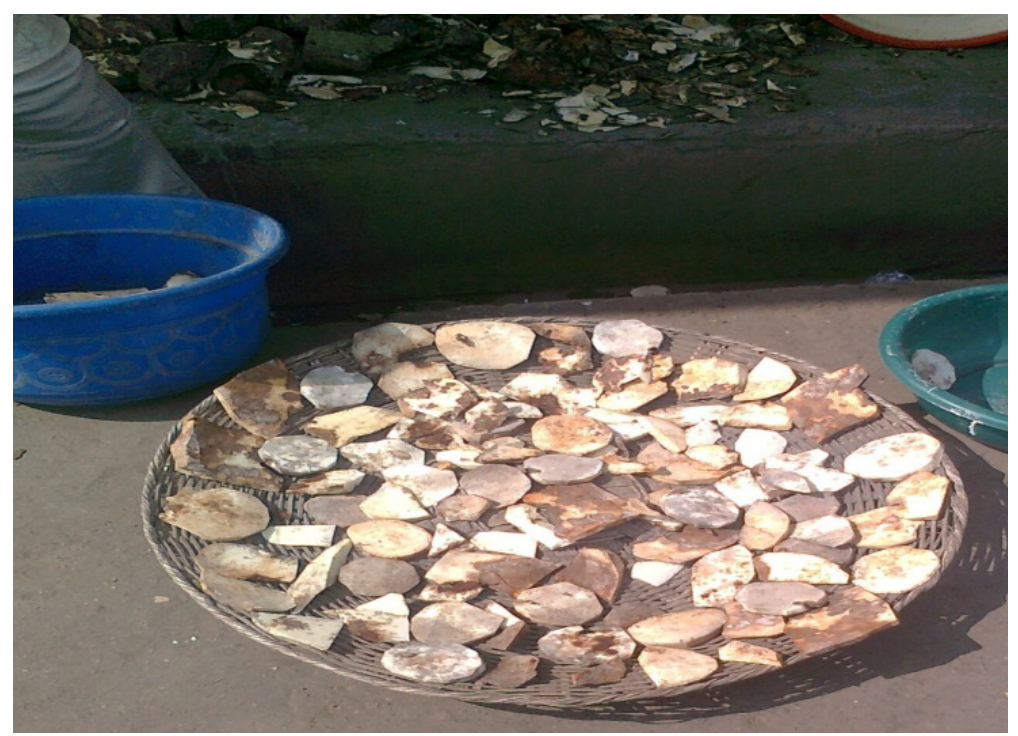

Figure 1. Typical rotten yam slices being dried for processing into yam chips

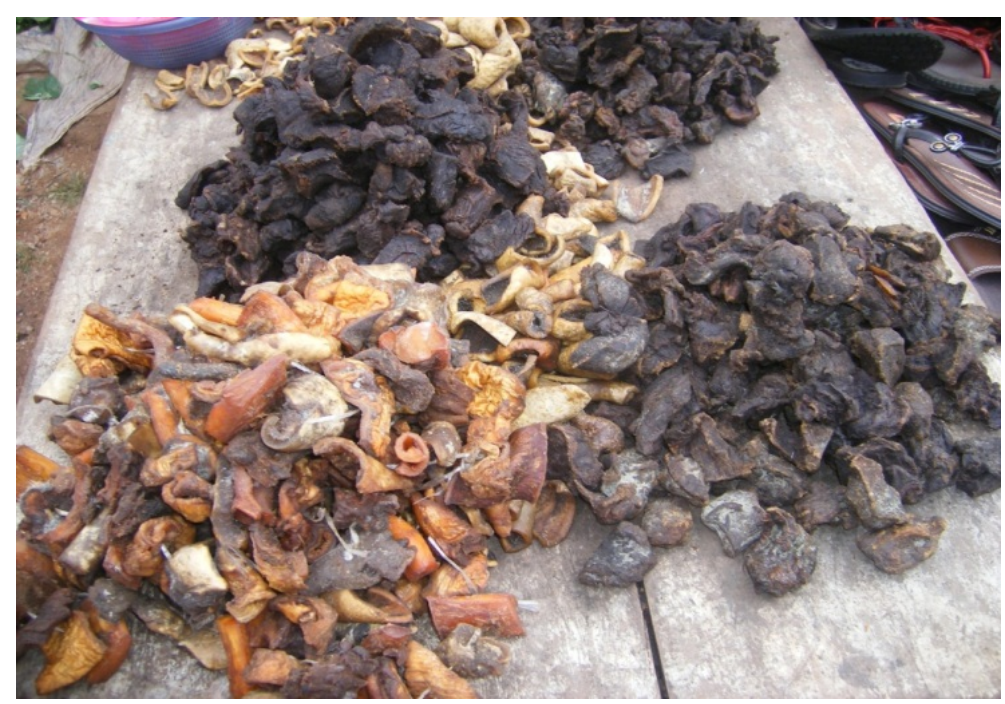

Figure 2. Aspergillus flavus contaminated dried meat displayed for sale in an open market in Nigeria 


\begin{tabular}{|c|c|c|c|c|c|c|c|}
\hline No & Aflatoxin & $\begin{array}{l}\text { Crop } \\
\text { contaminated }\end{array}$ & Location & $\begin{array}{l}\text { Frequency of } \\
\text { contamination }\end{array}$ & $\begin{array}{l}\text { Range of } \\
\text { concentration } \\
(\mu \mathrm{g} / \mathrm{kg})\end{array}$ & $\begin{array}{l}\text { Mean } \\
\text { concentration } \\
(\mu \mathrm{g} / \mathrm{kg})\end{array}$ & Author \\
\hline 1. & $\mathrm{AFB}_{1}$ & Groundnut & $\begin{array}{l}\text { Northern } \\
\text { Nigeria }\end{array}$ & & $100-2000$ & & Darling, 1963 \\
\hline 2 & $\mathrm{AFB}_{1}$ & $\begin{array}{l}\text { Stored } \\
\text { groundnut }\end{array}$ & Zaria & $35 \%$ & $100-2000$ & & Peer, 1965 \\
\hline 3 & $\begin{array}{l}\mathrm{AFB} 1 \& \\
\mathrm{G} 1\end{array}$ & Palm wine & Ibadan & & & & $\begin{array}{l}\text { Bassir and } \\
\text { Adekunle, } \\
(1969)\end{array}$ \\
\hline 4 & $\mathrm{AFB}_{1}$ & Bitter leaf & Ibadan & & $>94$ & & Bassir, 1969 \\
\hline 5 & $\mathrm{AFB}_{1}$ & $\begin{array}{l}\text { Groundnut } \\
\text { Dried fish } \\
\text { Cereals } \\
\text { (Millet } \\
\text { SorghumRice) }\end{array}$ & \begin{tabular}{|l|} 
Savannah \\
and \\
Forest \\
regions \\
of \\
Nigeria \\
\end{tabular} & & $\begin{array}{l}>900 \\
600-700 \\
150-300\end{array}$ & & $\begin{array}{l}\text { Okonkwo and } \\
\text { Nwokolo, } \\
(1978)\end{array}$ \\
\hline 6 & $\mathrm{AFB}_{1}$ & Sorghum & $\begin{array}{l}\text { Northern } \\
\text { Nigeria }\end{array}$ & & $<20$ & & Dada, 1978 \\
\hline 7 & $\begin{array}{l}\mathrm{AFB}_{1} \\
\mathrm{AFG}_{1}\end{array}$ & Sorghum & \begin{tabular}{|l|} 
Zaria \\
\end{tabular} & $\begin{array}{l}8 / 8 \\
8 / 8 \\
\end{array}$ & $\begin{array}{l}30.32-211.20 \\
2.40-208.00\end{array}$ & & $\begin{array}{l}\text { Uraih and } \\
\text { Ogbadu, (1982) }\end{array}$ \\
\hline 8 & $\begin{array}{l}\mathrm{AFB}_{1} \\
\mathrm{AFB}_{2} \\
\mathrm{AFG}_{1} \\
\mathrm{AFG}_{2}\end{array}$ & $\begin{array}{l}\text { Sera of } \\
\text { Primary liver } \\
\text { cell } \\
\text { carcinoma } \\
\text { patients } \\
\end{array}$ & Zaria & $\begin{array}{l}20 / 20 \\
20 / 20 \\
20 / 20 \\
20 / 20\end{array}$ & $\begin{array}{l}0.009-0.331 \\
0.030-0.278 \\
0.013-0.334 \\
0.005-0.146\end{array}$ & $\begin{array}{l}0.069 \\
0.095 \\
0.083 \\
0.040\end{array}$ & $\begin{array}{l}\text { Onyemelukwe } \\
\text { et al. (1982) }\end{array}$ \\
\hline 9 & $\mathrm{AFB}_{1}$ & \begin{tabular}{|l|} 
Groundnut \\
G/Nut oil \\
Cottonseed \\
oil \\
\end{tabular} & Zaria & & $\begin{array}{l}0-600 \\
>98 \\
>65\end{array}$ & & $\begin{array}{l}\text { Abalaka and } \\
\text { Elegbede, } 1982\end{array}$ \\
\hline 10 & $\mathrm{AFB}_{1}$ & \begin{tabular}{|l} 
Poultry feed \\
made of \\
groundnut \\
cake \\
(aflatoxicosis \\
in pecking \\
ducklings) \\
\end{tabular} & $\begin{array}{l}\text { Ogun } \\
\text { State }\end{array}$ & & & 3000 & $\begin{array}{l}\text { Ikwuegbu, } \\
\text { (1984) }\end{array}$ \\
\hline 11 & $\mathrm{AFB}_{1}$ & $\begin{array}{l}\text { Livestock } \\
\text { rations }\end{array}$ & Vom & & $4-340$ & & $\begin{array}{l}\text { Gbodi et } \\
\text { al.(1984) }\end{array}$ \\
\hline 12 & $\mathrm{AFB}_{1}$ & Millet beer & Jos & & & & $\begin{array}{l}\text { Okoye and } \\
\text { Ekpenyong, } \\
(1984)\end{array}$ \\
\hline 13 & $\mathrm{AFB}_{1}$ & Poultry feeds & \begin{tabular}{|l|}
$\begin{array}{l}\text { Southern } \\
\text { Nigeria }\end{array}$ \\
\end{tabular} & $69 / 120$ & $0.57-2.55$ & & $\begin{array}{l}\text { Oyejide et al. } \\
(1987)\end{array}$ \\
\hline 14 & $\mathrm{AFB}_{1}$ & Maize & Plateau & $27 / 6421 / 64$ & $\begin{array}{llll}0-960 & 0-543 & 0-\end{array}$ & $0.27-372.49$ & Gbodi, (1986) \\
\hline
\end{tabular}




\begin{tabular}{|c|c|c|c|c|c|c|c|}
\hline No & Aflatoxin & $\begin{array}{l}\text { Crop } \\
\text { contaminated }\end{array}$ & Location & $\begin{array}{l}\text { Frequency of } \\
\text { contamination }\end{array}$ & $\begin{array}{l}\text { Range of } \\
\text { concentration } \\
(\mu \mathrm{g} / \mathrm{kg})\end{array}$ & $\begin{array}{l}\text { Mean } \\
\text { concentration } \\
(\mu \mathrm{g} / \mathrm{kg})\end{array}$ & Author \\
\hline & $\begin{array}{l}\mathrm{AFB}_{2} \\
\mathrm{AFG}_{1} \\
\mathrm{AFG}_{2}\end{array}$ & & State & $5 / 643 / 64$ & $83.330-23.53$ & $\begin{array}{l}1.5-113.2 \\
20 \\
20.92-13.33\end{array}$ & \\
\hline 15 & $\begin{array}{l}\mathrm{AFB}_{1} \\
\mathrm{AFB}_{2} \\
\mathrm{AFG}_{1} \\
\mathrm{AFG}_{2} \\
\end{array}$ & Acha & $\begin{array}{l}\text { Plateau } \\
\text { State }\end{array}$ & $\begin{array}{l}4 / 242 / 240 / 24 \\
0 / 24\end{array}$ & $0-200-12$ & & Gbodi, (1986) \\
\hline 16 & $\begin{array}{l}\mathrm{AFB}_{1} \\
\mathrm{AFB} \\
\mathrm{AFG} \\
\mathrm{AFG}_{2}\end{array}$ & Cottonseed & $\begin{array}{l}\text { Plateau } \\
\text { State }\end{array}$ & $\begin{array}{l}3 / 8 \\
3 / 8 \\
2 / 8 \\
1 / 8 \\
\end{array}$ & $\begin{array}{l}0-271 \\
0-36.6 \\
0-183 \\
0-9.1\end{array}$ & $\begin{array}{l}52.25 \\
24.85 \\
38.13 \\
1.14 \\
\end{array}$ & Gbodi, (1986) \\
\hline 17 & AFs & Millet beer & & $10 / 10$ & $500-5000$ & & $\begin{array}{l}\text { Obasi et al. } \\
(1987)\end{array}$ \\
\hline 18 & $\mathrm{AFB}_{1}$ & Peanut cake & Nigeria & $\begin{array}{l}18 / 20 \\
29 / 29\end{array}$ & \begin{tabular}{|l}
$20-455$ \\
$13-2824$
\end{tabular} & 236.69 & $\begin{array}{l}\text { Akano \& } \\
\text { Atanda, } 1990 \\
\text { Ezekiel et al. } \\
\text { 2012c } \\
\end{array}$ \\
\hline 19 & $\mathrm{AFB}_{1}$ & $\begin{array}{l}\text { Cowpea } \\
\text { Maize } \\
\text { Millet } \\
\text { Rice } \\
\text { Sorghum } \\
\text { Cottonseed } \\
\text { Groundnut } \\
\text { Groundnut } \\
\text { oil } \\
\text { Melon seed } \\
\text { Palm kernel }\end{array}$ & $\begin{array}{l}\text { North } \\
\text { and } \\
\text { South } \\
\\
\\
\text { Lagos }\end{array}$ & $\begin{array}{l}3 / 268 \\
81 / 281 \\
10 / 275 \\
13 / 279 \\
22 / 318 \\
17 / 28 \\
414 / 634 \\
56 / 57 \\
22 / 30 \\
41 / 55\end{array}$ & $\begin{array}{l}0-48 \\
0-1250 \\
0-160 \\
0-40 \\
0-40 \\
\\
0-8000 \\
0-40 \\
0-53\end{array}$ & \begin{tabular}{|l}
31.6 \\
$74-218$ \\
42 \\
5 \\
5 \\
105 \\
$151-767$ \\
9.5 \\
19
\end{tabular} & $\begin{array}{l}\text { Opadokun, } \\
1992 \\
\text { (Samples } \\
\text { collected from } \\
1962-1985)\end{array}$ \\
\hline 20 & Total AF & $\begin{array}{l}\text { Restaurant } \\
\text { dishes (gari, } \\
\text { bean with } \\
\text { soup) } \\
\text { Dried okra } \\
\text { Dried pepper } \\
\end{array}$ & Nsukka & $17 / 17$ & $31.20-268.32$ & & $\begin{array}{l}\text { Obidoa and } \\
\text { Gugnani, (1992) }\end{array}$ \\
\hline 21 & Total AF & \begin{tabular}{|l} 
Corn \\
Corn cake \\
Corn roll \\
snack \\
\end{tabular} & $\begin{array}{l}\text { Western } \\
\text { Nigeria }\end{array}$ & & $\begin{array}{l}25-777 \\
15-1070 \\
10-160\end{array}$ & $\begin{array}{l}200 \\
233 \\
55\end{array}$ & $\begin{array}{l}\text { Adebajo et al. } \\
\text { (1994) }\end{array}$ \\
\hline 22 & $\mathrm{AFB}_{1}$ & $\begin{array}{l}\text { Red hot chili } \\
\text { pepper }\end{array}$ & $\begin{array}{l}\text { Western } \\
\text { Nigeria }\end{array}$ & & $>2.2$ & & $\begin{array}{l}\text { Adegoke } \text { et al. } \\
\text { (1996) }\end{array}$ \\
\hline 23 & $\begin{array}{l}\mathrm{AFB}_{1} \\
\mathrm{AFB}_{2}\end{array}$ & Maize & $\begin{array}{l}\text { Niger } \\
\text { State }\end{array}$ & $144 / 288$ & $\begin{array}{l}234-908 \\
234\end{array}$ & & Tijani, (2005) \\
\hline
\end{tabular}




\begin{tabular}{|c|c|c|c|c|c|c|c|}
\hline No & Aflatoxin & $\begin{array}{l}\text { Crop } \\
\text { contaminated }\end{array}$ & Location & $\begin{array}{l}\text { Frequency of } \\
\text { contamination }\end{array}$ & $\begin{array}{l}\text { Range of } \\
\text { concentration } \\
(\mu \mathrm{g} / \mathrm{kg})\end{array}$ & $\begin{array}{l}\text { Mean } \\
\text { concentration } \\
(\mu \mathrm{g} / \mathrm{kg})\end{array}$ & Author \\
\hline 24 & $\mathrm{AFM}_{1}$ & $\begin{array}{l}\text { Human milk } \\
\text { Cow milk } \\
\text { Ice cream }\end{array}$ & $\begin{array}{l}\text { Abeokuta } \\
\& \text { Odeda } \\
\text { local } \\
\text { Govt. } \\
\text { Nigeria }\end{array}$ & $\begin{array}{l}5 / 28 \\
3 / 22 \\
2 / 6\end{array}$ & & $\begin{array}{l}4.00(\mu \mathrm{g} / \mathrm{l}) \\
3.02(\mu \mathrm{g} / \mathrm{l}) \\
2.23(\mu \mathrm{g} / \mathrm{l})\end{array}$ & $\begin{array}{l}\text { Atanda et al., } \\
2007\end{array}$ \\
\hline 25 & $\mathrm{AFB}_{1}$ & Mouldy rice & $\begin{array}{l}\text { Niger } \\
\text { State } \\
\end{array}$ & $97 / 196$ & $0-1642$ & 200.19 & $\begin{array}{l}\text { Makun et al. } \\
(2007)\end{array}$ \\
\hline 26 & $\mathrm{AFB}_{1}$ & $\begin{array}{l}\text { Mouldy } \\
\text { sorghum }\end{array}$ & $\begin{array}{l}\text { Niger } \\
\text { State }\end{array}$ & $91 / 168$ & $0-1164$ & 199.51 & $\begin{array}{l}\text { Makun et al. } \\
(2009)\end{array}$ \\
\hline 27 & $\begin{array}{l}\mathrm{AFM}_{1} \\
\mathrm{AFB}_{1}\end{array}$ & $\begin{array}{l}\text { Powdered } \\
\text { milk } \\
\text { Bean } \\
\text { Wheat } \\
\end{array}$ & $\begin{array}{l}\text { Lagos } \\
\text { Minna } \\
\text { Minna }\end{array}$ & $\begin{array}{l}7 / 100 \\
29 / 50 \\
27 / 50\end{array}$ & $\mid \begin{array}{l}0-0.41 \\
0-137.6 \\
0-198.4\end{array}$ & $\begin{array}{l}0.016-0.325 \\
59.29 \\
85.56\end{array}$ & $\begin{array}{l}\text { Makun et al. } \\
(2010)\end{array}$ \\
\hline 28 & $\begin{array}{l}\mathrm{AFB}_{1} \\
\mathrm{AFB}_{2} \\
\mathrm{AFG}_{1} \\
\mathrm{AFG}_{2} \\
\text { Total } \mathrm{AF} \\
\end{array}$ & Rice & Minna & $\begin{array}{l}21 / 21 \\
21 / 21 \\
21 / 21 \\
19 / 21 \\
21 / 21 \\
\end{array}$ & \begin{tabular}{|l|}
$4.1-309$ \\
$1.3-24.2$ \\
$5.5-76.8$ \\
$3.6-44.4$ \\
$27.7-371.9$ \\
\end{tabular} & \begin{tabular}{|l}
37.2 \\
8.3 \\
22.1 \\
14.7 \\
82.5 \\
\end{tabular} & $\begin{array}{l}\text { Makun et al. } \\
(2011)\end{array}$ \\
\hline 29 & AFB1 & Melon seed & \begin{tabular}{|l|} 
Western \\
Nigeria
\end{tabular} & $30 / 120$ & $2.3-15.4$ & & $\begin{array}{l}\text { Bankole et al. } \\
2004\end{array}$ \\
\hline 30 & Total AF & Maize & $\begin{array}{l}\text { Western } \\
\text { Nigeria }\end{array}$ & $20 / 103$ & 3-138 & & \begin{tabular}{|l|} 
Bankole and \\
Mabekoje, 2004 \\
\end{tabular} \\
\hline 31 & $\begin{array}{l}\mathrm{AFB}_{1} \\
\mathrm{AFB}_{2} \\
\mathrm{AFG}_{1}\end{array}$ & Fonio millet & $\begin{array}{l}\text { Plateau } \\
\text { State, } \\
\text { Nigeria }\end{array}$ & $\begin{array}{l}13 / 16 \\
4 / 16 \\
4 / 16 \\
\end{array}$ & $\begin{array}{l}0.08-1.4 \\
0.07-0.1 \\
0.2-2\end{array}$ & $\begin{array}{l}0.4 \\
0.08 \\
0.6 \\
\end{array}$ & $\begin{array}{l}\text { Ezekiel et al, } \\
2012\end{array}$ \\
\hline 32 & $\begin{array}{l}\mathrm{AFB}_{1} \\
\mathrm{AFB}_{2} \\
\mathrm{AFG}_{1} \\
\mathrm{AFG}_{2}\end{array}$ & $\begin{array}{l}\text { Commercial } \\
\text { poultry feeds }\end{array}$ & Nigeria & $\begin{array}{l}44 / 58 \\
29 / 58 \\
35 / 58 \\
6 / 58\end{array}$ & \begin{tabular}{|l|}
$6-1067$ \\
$10-114$ \\
$8-235$ \\
$10-20$
\end{tabular} & \begin{tabular}{|l}
198 \\
34 \\
45 \\
13
\end{tabular} & $\begin{array}{l}\text { Ezekiel et al. } \\
2012 b\end{array}$ \\
\hline 33 & $\mathrm{AFB}_{1}$ & $\begin{array}{l}\text { Rice } \\
\text { Beans } \\
\text { Cassava flour } \\
\text { Semovita } \\
\text { Yam } \\
\text { Wheat meal } \\
\text { Maize } \\
\text { "Gari" } \\
\end{array}$ & $\begin{array}{l}\text { Ogun } \\
\text { State, } \\
\text { Nigeria }\end{array}$ & $\begin{array}{l}19 / 21 \\
15 / 17 \\
3 / 4 \\
2 / 6 \\
6 / 7 \\
2 / 3 \\
3 / 3 \\
13 / 18 \\
\end{array}$ & $\begin{array}{l}\text { *nd }-0.30 \\
\text { nd- } 0.89 \\
\text { nd- } 0.07 \\
\text { nd- } 0.17 \\
\text { nd- } 0.27 \\
\text { nd- }-0.06 \\
0.11-0.20 \\
\text { nd- } 0.69\end{array}$ & $\begin{array}{l}0.14 \\
0.15 \\
0.05 \\
0.09 \\
0.14 \\
0.04 \\
0.16 \\
0.25 \\
\end{array}$ & $\begin{array}{l}\text { Adejumo et } \\
\text { al.,2012. } \\
\text { Unpublished. } \\
\text { Submitted to } \\
\text { Food\& } \\
\text { Chemical } \\
\text { Toxicology }\end{array}$ \\
\hline
\end{tabular}




\begin{tabular}{|c|c|c|c|c|c|c|c|}
\hline No & Aflatoxin & $\begin{array}{l}\text { Crop } \\
\text { contaminated }\end{array}$ & Location & $\begin{array}{l}\text { Frequency of } \\
\text { contamination }\end{array}$ & \begin{tabular}{|l|} 
Range of \\
concentration \\
$(\mu \mathrm{g} / \mathrm{kg})$
\end{tabular} & $\begin{array}{l}\text { Mean } \\
\text { concentration } \\
(\mu \mathrm{g} / \mathrm{kg})\end{array}$ & Author \\
\hline & $\mathrm{AFM}_{1}$ & $\begin{array}{l}\text { Breast milk of } \\
\text { lactating } \\
\text { mothers }\end{array}$ & $\begin{array}{l}\text { Ogun } \\
\text { State, } \\
\text { Nigeria }\end{array}$ & $41 / 50$ & $\begin{array}{l}\text { nd- } \\
92.14(\mathrm{ng} / \mathrm{l})\end{array}$ & 15.00(ng/l) & \\
\hline 34 & $\begin{array}{l}\mathrm{AFB}_{1} \\
\mathrm{AFB}_{2} \\
\mathrm{AFG}_{1} \\
\mathrm{AFG}_{2} \\
\text { Total AF }\end{array}$ & $\begin{array}{l}\text { Red hot chili } \\
\text { pepper }\end{array}$ & Minna & $\begin{array}{l}12 / 20 \\
12 / 20 \\
12 / 20 \\
12 / 20 \\
12 / 20\end{array}$ & $\begin{array}{l}0.05-12.4 \\
0.05-4.95 \\
0.50-10.0 \\
0.55-3.20 \\
0.85-19.45\end{array}$ & $\begin{array}{l}2.21 \\
0.89 \\
0.58 \\
0.39 \\
5.85\end{array}$ & $\begin{array}{l}\text { Unpublished. } \\
\text { Submitted to } \\
\text { Food Control. } \\
\text { Makun et al. } \\
(2012)\end{array}$ \\
\hline 35 & $\mathrm{AFM}_{1}$ & $\begin{array}{l}\text { Fresh milk } \\
\text { “Nono” } \\
\text { “Kindirmo" }\end{array}$ & Bida & $\begin{array}{l}10 / 10 \\
10 / 10 \\
10 / 10\end{array}$ & $\begin{array}{l}0.41-0.96 \\
0.25-2.51 \\
015-0.70\end{array}$ & & $\begin{array}{l}\text { Unpublished } \\
\text { Okeke, (2012) }\end{array}$ \\
\hline 36 & $\begin{array}{l}\mathrm{AFB}_{1} \\
\mathrm{AFB}_{2}\end{array}$ & Maize & $\begin{array}{l}\text { Niger } \\
\text { State }\end{array}$ & $\begin{array}{l}59 / 95 \\
67 / 95\end{array}$ & \begin{tabular}{|l|}
$4.0-74.4$ \\
$8.0-79.2$
\end{tabular} & $\begin{array}{l}19.58 \\
30.10\end{array}$ & $\begin{array}{l}\text { Unpublished } \\
\text { Muhammad, } \\
2012\end{array}$ \\
\hline 37 & $\begin{array}{l}\mathrm{AFB}_{1} \\
\mathrm{AFB}_{2}\end{array}$ & Rice & $\begin{array}{l}\text { Kaduna } \\
\text { State }\end{array}$ & $\begin{array}{l}64 / 86 \\
41 / 86\end{array}$ & $\begin{array}{l}4-292 \\
0.4-27.2\end{array}$ & $\begin{array}{l}157.34 \\
5.17\end{array}$ & $\begin{array}{l}\text { Unpublished } \\
\text { Olorunmowaju, } \\
2012\end{array}$ \\
\hline 38 & $\begin{array}{l}\mathrm{AFB}_{1} \\
\mathrm{AFB}_{2}\end{array}$ & Groundnut & $\begin{array}{l}\text { Niger } \\
\text { State }\end{array}$ & $\begin{array}{l}72 / 82 \\
61 / 82\end{array}$ & \begin{tabular}{|l|}
$4-188$ \\
$0.40-38.4$
\end{tabular} & $\begin{array}{l}53.06 \\
8.08\end{array}$ & $\begin{array}{l}\text { Unpublished } \\
\text { Ifeji, } 2012\end{array}$ \\
\hline
\end{tabular}

*nd $=$ Not detected

Table 1. Incidence of Aflatoxins in Nigerian Foods and Feeds

\begin{tabular}{|c|c|c|c|c|c|c|c|c|}
\hline S/No & Fungi & Mycotoxin & $\begin{array}{l}\text { Commodity } \\
\text { contaminate }\end{array}$ & Location & \begin{tabular}{|l}
$\begin{array}{l}\text { Frequency of } \\
\text { contami- } \\
\text { nation }\end{array}$ \\
\end{tabular} & $\begin{array}{l}\text { Concen- } \\
\text { tration } \\
\text { range } \\
(\mu \mathrm{g} / \mathrm{kg})\end{array}$ & $\begin{array}{l}\text { Mean } \\
\text { concentr- } \\
\text { ation }(\mu \mathrm{g} / \mathrm{kg})\end{array} \mid$ & Author \\
\hline 1 & Sphacelia sorghi & \begin{tabular}{|l} 
Dihydro- \\
ergosine
\end{tabular} & Guinea corn & $\begin{array}{l}\text { Northern } \\
\text { Nigeria }\end{array}$ & & & & Mantle (1968) \\
\hline 2 & A.flavus & $\begin{array}{l}\text { *Palmotoxin Bo } \\
\text { and Go }\end{array}$ & Palm wine & Ibadan & & & & $\begin{array}{l}\text { Bassir and } \\
\text { Adekunle, } \\
\text { (1969) }\end{array}$ \\
\hline 3 & $\begin{array}{l}\text { Aspergillius, } \\
\text { Fusarium, } \\
\text { Penicillium, } \\
\text { Phoma, } \\
\text { Alternaria, } \\
\text { Curvularia, } \\
\text { Chaetomium } \\
\text { and } \\
\text { Helminthospori } \\
\text { um }\end{array}$ & $\begin{array}{l}\text { ochratoxins A, } \\
\text { B and C, } \\
\text { sterigmatocysti } \\
n \text { and } \\
\text { zearalenone }\end{array}$ & Guinea corn & $\begin{array}{l}\text { Zaria, } \\
\text { Nigeria }\end{array}$ & & & & $\begin{array}{l}\text { Elegbede, } \\
\text { (1978), }\end{array}$ \\
\hline
\end{tabular}




\begin{tabular}{|c|c|c|c|c|c|c|c|c|}
\hline S/No & Fungi & Mycotoxin & $\begin{array}{l}\text { Commodity } \\
\text { contaminate }\end{array}$ & Location & \begin{tabular}{|l} 
Frequency of \\
contami- \\
nation
\end{tabular} & $\begin{array}{l}\text { Concen- } \\
\text { tration } \\
\text { range } \\
(\mu \mathrm{g} / \mathrm{kg})\end{array}$ & $\begin{array}{l}\text { Mean } \\
\text { concentr- } \\
\text { ation }(\mu \mathrm{g} / \mathrm{kg})\end{array}$ & Author \\
\hline & $\begin{array}{l}\text { Collectotrichum, } \\
\text { Pericona, } \\
\text { Rhizopus, } \\
\text { Mucor, } \\
\text { Trichotecium, } \\
\text { Cephalosporium }\end{array}$ & & & & & & & \\
\hline 4 & \begin{tabular}{|l|} 
Collectrotrichu \\
m, Curvularia, \\
Fusarium, \\
Mucor, Phoma, \\
Rhizopus, \\
Helminthospori \\
um, Penicillium \\
and \\
Trichoderma \\
apart from the \\
Aspergillius
\end{tabular} & $\begin{array}{l}\text { Patulin and } \\
\text { Zearalenone }\end{array}$ & Guinea corn & $\begin{array}{l}\text { Northern } \\
\text { Nigeria }\end{array}$ & & & & Salifu 1978 \\
\hline 5 & $\begin{array}{l}\text { Aspwegillus } \\
\text { spp, Phoma } \\
\text { sorghina, } \\
\text { Fusarium } \\
\text { semitectum, } \\
\text { Fusarium } \\
\text { moniliforme } \\
\text { and F.equiseti }\end{array}$ & $\begin{array}{l}\text { two } \\
\text { unidentified } \\
\text { metabolites }\end{array}$ & Guinea corn & $\begin{array}{l}\text { Bakura, } \\
\text { Kano, } \\
\text { Samaru } \\
\text { and } \\
\text { Mokwa. }\end{array}$ & & & & Dada, (1979) \\
\hline 6 & & Zearalenone & $\begin{array}{l}\text { Native Beer } \\
\text { (Burukutu) }\end{array}$ & $\begin{array}{l}\text { Jos, } \\
\text { Plateau } \\
\text { State }\end{array}$ & & & & Okoye 1986 \\
\hline 7 & & \begin{tabular}{|l|} 
ochratoxins \\
zearalenone, \\
vomitoxin, T-2 \\
toxin and \\
moniliform
\end{tabular} & $\begin{array}{l}\text { Cocoa, } \\
\text { groundnut, } \\
\text { palm kernel, } \\
\text { maize, yam, }\end{array}$ & $\begin{array}{l}\text { Northern } \\
\text { and } \\
\text { Southern } \\
\text { Nigeria }\end{array}$ & & & & Okoye (1992) \\
\hline 8 & & \begin{tabular}{|l|} 
Nivalenol, \\
fusarenon-X \\
and HT-2 \\
toxin, \\
deoxynivalenol \\
and T-2 toxin
\end{tabular} & Maize & Jos district & & & & Okoye (1993) \\
\hline 9 & $\begin{array}{l}\text { Botryodiplodia } \\
\text { theobromae } \\
\text { Cladosporium } \\
\text { herbarum, } \\
\text { Diplodia sp. } \\
\text { Fusarium } \\
\text { moniliforme }\end{array}$ & $\begin{array}{l}\text { Ochratoxin A } \\
\text { and } \\
\text { zearalenone }\end{array}$ & Kola nut & $\begin{array}{l}\text { South } \\
\text { Western } \\
\text { Nigeria }\end{array}$ & & & & $\begin{array}{l}\text { Adebajo and } \\
\text { Popoola (2003) }\end{array}$ \\
\hline
\end{tabular}




\begin{tabular}{|c|c|c|c|c|c|c|c|c|}
\hline $\mathrm{S} / \mathrm{No}$ & Fungi & Mycotoxin & $\begin{array}{l}\text { Commodity } \\
\text { contaminate }\end{array}$ & Location & $\mid \begin{array}{l}\text { Frequency of } \\
\text { contami- } \\
\text { nation }\end{array}$ & $\begin{array}{l}\text { Concen- } \\
\text { tration } \\
\text { range } \\
(\mu \mathrm{g} / \mathrm{kg})\end{array}$ & $\begin{array}{l}\text { Mean } \\
\text { concentr- } \\
\text { ation }(\mu \mathrm{g} / \mathrm{kg})\end{array}$ & Author \\
\hline & \begin{tabular}{|l} 
and F. \\
oxysporum, \\
Aspergillus \\
clavatus, A. \\
flavus Link, A. \\
niger, A. \\
ochraceus, A. \\
parasiticus and \\
A. tamarii \\
Penicillium \\
digitatum, P. \\
funiculosum, \\
Penicillium sp, \\
Rhizomucor \\
pusillus and \\
Rhizopus \\
arrhizus \\
Aspergillus \\
,Penicillium, \\
Botryodiplodia, \\
Cladosporium \\
and Rhizopus \\
species
\end{tabular} & & & & & & & \\
\hline 10 & \begin{tabular}{|l} 
Aspergillus and \\
Fusarium \\
species
\end{tabular} & $\begin{array}{l}\text { Aflatoxins and } \\
\text { Fumonisins }\end{array}$ & Maize & $\begin{array}{l}\text { South } \\
\text { western } \\
\text { Nigeria }\end{array}$ & & & & $\begin{array}{l}\text { Bankole and } \\
\text { Mabekoje } \\
(2004)\end{array}$ \\
\hline 11 & & $\begin{array}{l}\text { Aflatoxins, } \\
\text { ochratoxins } \\
\text { and fumonisins }\end{array}$ & $\begin{array}{l}\text { Cereals, } \\
\text { spices, } \\
\text { stockfish and } \\
\text { milk and } \\
\text { milk } \\
\text { products }\end{array}$ & $\begin{array}{l}\text { South } \\
\text { western } \\
\text { Nigeria }\end{array}$ & & & & $\begin{array}{l}\text { Ogunbanwo } \\
(2005)\end{array}$ \\
\hline 12 & & Ochratoxins & Cocoa & $\begin{array}{l}\text { South } \\
\text { Western } \\
\text { Nigeria }\end{array}$ & & & & $\begin{array}{l}\text { Aroyeun and } \\
\text { jayeola (2005) }\end{array}$ \\
\hline 13 & \begin{tabular}{|l|} 
Aspergillus, \\
Penicillium, \\
Fusarium, \\
Alternaria, \\
Mucor, \\
Rhizopus, \\
Trichoderma, \\
Curvularia, \\
Helminthospori \\
um and \\
Cladosporium. \\
Others include \\
Arthrinium, \\
Syncephalastru
\end{tabular} & $\begin{array}{l}\text { Ochratoxin A } \\
\text { and } \\
\text { zearalenone }\end{array}$ & $\begin{array}{l}\text { Guinea corn } \\
\text { and rice }\end{array}$ & & Niger state & & & Makun (2007) \\
\hline
\end{tabular}




\begin{tabular}{|c|c|c|c|c|c|c|c|c|}
\hline S/No & Fungi & Mycotoxin & $\begin{array}{l}\text { Commodity } \\
\text { contaminate }\end{array}$ & Location & $\begin{array}{l}\text { Frequency of } \\
\text { contami- } \\
\text { nation }\end{array}$ & $\begin{array}{l}\text { Concen- } \\
\text { tration } \\
\text { range } \\
(\mu \mathrm{g} / \mathrm{kg})\end{array}$ & \begin{tabular}{|l|} 
Mean \\
concentr- \\
ation $(\mu \mathrm{g} / \mathrm{kg})$
\end{tabular} & Author \\
\hline & $\begin{array}{l}\text { m, Geotrichum, } \\
\text { Bipolaris, } \\
\text { Rhodotorula, } \\
\text { Cryptococcus, } \\
\text { orula, } \\
\text { Chrysosporium, } \\
\text { Collectitrichum, } \\
\text { Scopulariopsis, } \\
\text { Gilocladium } \\
\text { and Nocardia } \\
\end{array}$ & & & & & & & \\
\hline 14 & & \begin{tabular}{|l|} 
3- \\
Nitropropionic \\
acid \\
Averufanin \\
Averufin \\
Beauvericin \\
Cyclosporin A \\
Emodin \\
Equisetin \\
Kojic acid \\
Nidurufin \\
Norsolorinic \\
acid \\
Ochratoxin A \\
Methlysterigm \\
atocystin \\
Sterigmatocystin \\
Versicolorin A \\
Versicolorin
\end{tabular} & Peanut cake & & & & \begin{tabular}{|l}
20 \\
0.2 \\
0.2 \\
1.0 \\
0.02 \\
0.2 \\
1.0 \\
5.0 \\
80 \\
0.4 \\
0.02 \\
4 \\
4 \\
2 \\
2 \\
0.2 \\
1.0
\end{tabular} & $\begin{array}{l}\text { Ezekiel et al., } \\
2012\end{array}$ \\
\hline
\end{tabular}

Table 2. Fungi and other Mycotoxins in Nigerian Foods

\section{Human and animal health implications of mycotoxins in Nigeria}

Mycoses and mycotoxicoses are the major ways in which human and animal health is affected due to infection with fungi and contamination with Mycotoxins. When this happens the implications are wide and span from health to economics. Mycosis means fungal infection of man or animals and range in its simplest form from growth that merely annoys the victim to a more complex form of life threatening invasion. Mycotoxicosis on the other hand could be defined as a disease outbreak that is commonly associated with the ingestion of mycotoxins or inhalation of spores produced by fungi. The appearance of mycotoxicoses symptoms depends on the level of contamination, length of exposure, type of mycotoxins, degree of combination with several other mycotoxins, individual differences, speciesspecific resistance, sex, pre-existing pathological and physiological status of the victim. The synergistic effects associated with several other factors such as genetics, diet, and interactions with other toxins have been poorly studied. Therefore, it is possible that vitamin deficiency, caloric deprivation, alcohol abuse and infectious disease status can all have 
compounded effects with mycotoxins. Mycotoxins have the potential for both acute and chronic health effects through ingestion, skin contact and inhalation. These toxins can enter the blood stream and lymphatic system and inhibit protein synthesis, damage macrophage systems, particle clearance of the lung and increase sensitivity to opportunistic infections. If symptoms appear within a short period of less than 7 days of contamination, it is termed "acute mycotoxicoses" but if the interval between contamination and appearance of the symptoms persist longer, it is termed "chronic mycotoxicoses". In acute cases, victim may die if adequate treatment measures are not taken whereas in chronic cases, the victim may live longer though with protracted illnesses.

\subsection{Human mycotoxicoses}

Mycotoxins have been detected in human foods and livestock feeds in Nigeria. Most often these mycotoxins are detected in deleterious levels compounded by synergistic interactions. Whenever these contaminated foods and feeds are ingested, the victim's physiological mechanisms attempt to metabolize the toxins especially the liver, kidneys and some specialized microorganisms in the gastrointestinal tract (Bankole and Adebanjo, 2003). On a world wide scale, relationships between mycotoxins and human illness have been clearly established. Aflatoxins have been shown to be involved with and to aggravate hepatitis B infection (JECFA 2001), while Fumonisins have been shown to be consistently responsible for oesophageal cancer in south Africa (Makaula et al. 1996). In Nigeria, documentation of Mycotoxin related human health problems is not extensive. However the few literature available show evidence of human mycotoxicoses and suspected mycotoxicoses related deaths. The death of some children who consumed mouldy Kulikuli (Groundnut cake) in Ibadan was suspected to be due to aflatoxicosis (Ikeorah and Okoye 2005). Aflatoxins have been found in the urine of liver disease patients in Zaria, in Blood in Southern Nigeria, in organs of children who died of kwashiorkor in Western Nigeria, and in human semen in Benin city (Onyemelukwe 1992, Oluyide 1993, Adegoke et al. 1996 and Oluwafemi 2005). Similarly Aflatoxin $\mathrm{M}_{1}$ has been found in breast milk and in the blood of umbilical cord of babies in the country (WHO 1997 and Adejumo et al. 2012). Doctors at the National hospital Abuja have reported post mortem examination link between the cause of death from liver cancer to Aflatoxin (Paul Jibrin - personal communication). Some Specialist Hospitals visited, gave reports of several mycological cases suspected to be synonymous with Aflatoxicoses, Ochratoxicoses, Zearalenone and Fumonisin toxicities. However, legislation on Medical Ethics in the country restrained us from accessing individual case files. In some of these hospitals, the cases were documented as fungal infections, mycoses and mycotoxicoses and because this area of study is not yet very popular, many Nigerians are not conversant with the words mycoses and mycotoxicoses (Idahor et al., 2010; Idahor and Ogara, 2010).

\subsection{Livestocks}

Mycotoxins are known to be consumed by livestock through contaminated feed ingredients. They are probably the causative agents or suspected contributing factors in farm animal diseases that cause great economic losses. Feed ingredients are any of the constituent 
nutrients of livestock ration. Some of the plants used in ration formulation like cereals, legumes, oil seeds, nuts and root crops are susceptible to mycotoxins contamination yet their deleterious effects are still a grey area. In livestock, mycotoxicoses cases are more severe in monogastric than ruminants due to the detoxifying capabilities of some rumen microorganisms. Young and pregnant animals are generally the most susceptible to mycotoxicoses. Under some conditions, the fungi may produce potent mycotoxins at levels that may adversely affect livestock production. At moderate levels, effects may appear initially with more obvious symptoms within a few days to several weeks of ingestion of the contaminated foods or feeds. Mycotoxins could possibly have pervasive yet subclinical effects on performance and health in ruminants that may be unnoticed. Performance losses of $5-10 \%$ are typical with consumption of mouldy feeds even in the absence of mycotoxins. On the other hand, mycotoxins contaminations increase production losses even when the mould is not readily visible. In horses, equine leukoencephalomalacia syndrome fatal mycotoxic disease occuring only in horses, donkeys and ponies is is characterized by the presence of liquefactive necrotic lesions in the white matter of the horse cerebrum. Other pathological changes of this disease include lethargy, head pressing, inappetence, convulsion and sudden death. There are few reported suspected cases of aflatoxicoses in horses associated with Penicillium purpurogenum in Vom, plateau state (Ocholi et al. 1992) and aflatoxicoses in Pecking ducklings in Ogun state.

Rabbits seem apparently unsusceptible to micro doses of mycotoxins especially when dosed orally for a relatively short period. Idahor et al. (2008a) observed gradual decrease in sperm production rates, final live weights, feed consumption and body weight gain concomitantly with increasing Fumonisin $\mathrm{B}_{1}$ concentration in diet. There were no evidences of mycotoxins (at $1.9 \mathrm{mg} / \mathrm{kg}$ ) diet crossing the placenta to cause developmental abnormalities in the foetuses examined at the first trimester. It was speculated that there might have been some damages on the physiological status of the rabbits and possibly gradual accumulation of the mycotoxins in the carcasses which might in turn pose residual health hazard to humans when consumed (Idahor et al. 2008b) . In a similar study, Ogunlade et al. (2004) reported sufficient evidences of carcinogenicity and toxicity at micro doses of 1650-1990 $\mu$ g Fumonisin $\mathrm{B}_{1}$ per $\mathrm{kg}$ diet. But there were no negative effects on the rabbit's blood cellular components, serum protein metabolism and serum enzymes activities. On the other hand, Ewuola et al. (2003) demonstrated that micro doses of Fumonisin $B_{1}$ can induce physiological and pathological damages in rabbits by reducing the feed intake with resultant effects on body weight gain.. Pregnant New Zealand White rabbits are speculated to be very sensitive to the toxic effects of Fumonisin $\mathrm{B}_{1}$ and that maternal toxicity was observed at daily gavage doses of $0.25 \mathrm{mg} / \mathrm{kg}$ body weight.

\section{Economic impact of mycotoxins in Nigeria}

Estimating the economic impact of mycotoxins require good data sets and expertise in the use of various economic impact assessment models. Both of these prerequisites generally are missing in Sub-Saharan Africa. Data which would form the baseline on the effects and related costs of mycotoxin contamination on human health are not available. Cost elements 
include mortality (the cost of productive capacity lost with premature death), the cost of morbidity( losses resulting from productivity loss, hospitalization and the costs of health care services both for public and private health Institutions). There is also the intangible cost of pain, suffering, anxiety, and reduction in the quality of life (Lubulwa and Davis, 1994). The data on the economic impact of mycotoxins on livestock which should include income losses due to mortality as well as those due to reductions in productivity, weight gain, and the yield of meat, milk and eggs, as well as those due to feed use inefficiency and increased susceptibility to disease are also not available in Nigeria. The economic losses associated with mycotoxin contamination are difficult to assess in a consistent and uniform way. The lack of information on the health costs and other economic losses from mycotoxin induced human illness is partly due to the difficulty of establishing cause-and-effect relationships between mycotoxins and the chronic diseases they are suspected of causing. For SubSaharan Africa, capacity building for economic impact assessment is urgent for both impact assessment and trade analysis. Baseline data do not exist in most of the countries including Nigeria, so comparisons often have no basis for normalization.

Even trade data are difficult to obtain. The trade data used in the gravity model by Otsuki et al. $(2001 a, b)$ to establish the baseline put African exports to the European Union in 1998 to be US\$ 472 million for dried fruit and nuts and US\$ 298 million for cereals, with the bulk of this trade occurring with France. These figures seem implausible, especially for cereals, given Africa's lack of competitiveness in this sector relative to Europe, and statistics from the United Nations COMTRADE database show that European imports from Africa are not large. Africa only exported to Europe approximately US\$ 104 million of dried fruit, US\$ 45 million of peanuts, US $\$ 27$ million for other edible nuts, and < US\$ 14 million for cereals and cereal products in 1998. Thus, the baseline against which economic impacts should be defined is at best fuzzy and often lacking (Leslie et al, 2008)

\subsection{Economic losses and impact on international trade}

The adverse economic effects attributed to mycotoxin contamination and losses are widely felt in all sectors of food production and particularly in agricultural commodities.

Mycotoxin contamination of agricultural commodities has considerable economic implications. Losses from rejected shipments and lower prices for inferior quality can devastate developing-country export markets.

Produce handlers are affected by restricted storage options, cost of testing produce lots and loss of end market locally. Companies incur higher costs due to higher product losses, monitoring costs and restricted end markets. Consumers who are the primary target in this chain end up paying higher prices due to increased monitoring at all levels of handling, and in extreme cases health problems due to consumption of contaminated products.

Costs to farmers include reduced income from outright food or feed losses and lower selling prices for contaminated commodities. The economic impact on livestock production includes mortality as well as reductions in productivity, weight gain, feed efficiency, 
fertility, ability to resist diseases and decrease in the quantity and quality of meat, milk and egg production.

Any economic costs must be weighed against the costs of preventing mycotoxins through better production, harvesting and storage practices. The latter costs are likely to be considerable. Member states of the African Groundnut Council (Gambia, Mali, Niger, Nigeria, Senegal, and Sudan) have calculated the annual cost of implementing a program to reduce mycotoxin contamination at US\$7.5 million. High levels of mycotoxins have been found in groundnuts and cereal grains in countries such as Gambia, Ghana, Guinea, Nigeria, Senegal, South Africa and indications of the magnitude of the problem.

Contamination of food by microbes and chemicals also has economic consequences due to rejection of exports and loss of credibility as trading partners. Capacity to implement effective food safety controls is of vital importance to agricultural and food exports from developing countries. For example, importing countries frequently require guarantees that minimum standards of hygiene have been applied in the manufacture of a food product and that food do not have excessive mycotoxins contamination. The exporting country must be able to comply with these requirements and demonstrate that compliance has been achieved. While basic scientific and technical infrastructure is clearly vital, administrative structures, management, financing and human capital are also important elements. Indeed, the experiences of many countries suggest that lack of efficient management or sustainable levels of resources can seriously compromise the effectiveness of food safety controls.

In Nigeria, regulatory agencies destroyed mycotoxin-contaminated foods worth more than US\$ 200000 in 2010.

Total aflatoxin (B1, B2, G1 and G2) content in ng/g of product can give an indication of product quality and can be used as a threshold for separating high, medium and low quality produce. This grading is used for pricing a premium (high quality) or a discount (poor quality) crop. The risk of spoilage is a function of factors including: the variety of crop, the time and method of harvest and storage, the storage temperature, the moisture content and the drying method prior to storage. Africa loses an estimate of sixty seven (\$67) million dollars annually from export rejects due to high levels of mycotoxins in food and agricultural produce coming from developed countries, including Nigeria. Over the years, Nigeria has received notifications from the European Union Rapid Alert System on export rejects originating from Nigeria.

\section{Possible intervention strategies/regulations for mycotoxins in Nigeria}

Nigeria is a country of marked ecological diversity and climatic contrasts with biophysical characteristics, agro-ecological zones and socio-economic conditions. (Aregheore 2005). Crop production in the country is dominated by cereal, root and tuber crops (Amujoyegbe 2012) The climatic conditions and agricultural practices therefore affect mycotoxin prevalence greatly in the different parts of Nigeria. Current research in the country shows that the prevalence of mycotoxins is all over Nigeria because they have been identified from 
various commodities from the various agro ecological zones. The complete elimination of mycotoxin contaminated commodities is not achievable hence good agricultural practices (GAP) represent a primary line of defense against contamination of cereals with mycotoxins, followed by the implementation of good manufacturing practices (GMP) during the handling, storage, processing, and distribution of cereals for human food and animal feed. (Codex alimentarius commissin, 2003). The achievement of mycotoxin reduction and control is dependent on the concerted actions of all actors along the food production and distribution chain. Multidisciplinary approaches are therefore critical (FOS, 2006). Any possible intervention strategies to reduce mycotoxins in Nigeria as elsewhere must begin from good Agricultural practices that involves both pre-harvest and post harvest stages followed by Good manufacturing practices (GMP), Risk Assessment for Mycotoxin Contamination and Good Storage Practices will then follow.

\subsection{Good agricultural practices}

Good Agricultural Practices is a collection of principles to apply for on-farm production and post-production processes that will result in safe and healthy food and non-food agricultural products. Activities to ensure good agricultural practices must be given a holistic approach to tackle the menace of mycotoxins. Efforts to mitigate mycotoxins contamination will be successful if all good agricultural principles are put in place by farmers in Nigeria.

\subsection{Soil}

Under good agricultural principles the type of land, soil conditions, soil management must not have any negative effect on the biodiversity. GAP also lead to soil productivity, availability, uptake of water and nutrients through enhanced soil biological activity. The soil organic matter replenished, losses of soil moisture, nutrients, and agrochemicals through erosion are reduced, and runoff and leaching into surrounding environment, sediments, nutrients movement, and mobility of livestock and associated species including predators, pests and biocontrol agents are brought under control.

\subsection{Pre-harvest interventions}

Crop management at the pre harvest stage is critical to mycotoxin reduction. Planting, pre harvest and postharvest strategies for a particular crop depends on the climatic conditions of that particular year, taking into account the local crops, and traditional production conditions for that particular country or region (CAC RCP 51 2003). Wet weather either during flowering or at harvest is also a major risk factor (Negedu, et al, 2011). In general, pre harvest management must ensure that insect damage and fungal infection in the vicinity of the crop must be minimized by proper use of registered insecticides, fungicides, herbicides and making sure that mechanical injury to plants during cultivation is minimized. Seed varieties that are resistant to insect pest and diseases should be procured. Irrigation is a valuable method of reducing plant stress that is sometimes responsible for mycotoxin development crops in some growing situations. Crop rotation should be developed and 
maintained but crops identified to be susceptible to toxigenic moulds should not be used in rotation with each other. Grains should be harvested at full maturity, cleaned and infected seeds removed using procedures such as gravity table. They should be promptly dried to low moisture levels $(13 \%)$ before storage where applicable. Moisture levels of the crop should be determined at several spots during harvest, immediately after harvest, before and during storage in barns or silos. Sampling for such test should be as representative of the lot as possible.

\subsection{Storage}

Storage is a critical stage where infection and mycotoxin accumulation occur. Care must be taken to store grains that are wholesome and apparently healthy. Prestorage treatment or handling should take care of certain basic issues. Winnowing grains at harvest or later should be done to remove shriveled small grains which may contain more zearalenone than healthy normal grains. Harvesting and storage conditions should be documented with daily temperature and humidity checks. Wet grains provide suitable environment for mould growth and should not be piled up for a long time to reduce the risk of fugal growth. Bagged commodities should be stored on pallets. Storage facilities meant to exclude mold growth should include dry and well-ventilated structures, provide protection from rain, drainage of ground water; prevent entry of rodents and birds, and minimum temperature fluctuations. A temperature rise of $2-3^{\circ} \mathrm{C}$ may indicate microbial growth and/or insect infestation. Where this happens, the apparently infected portions of the grain should be separated and sent for analysis. Mycotoxin levels in grain should be monitored using appropriate sampling and testing programs.

\subsection{Transportation}

Transport containers should be dry and free of visible fungal growth, insects and any contaminated material they should be cleaned and disinfected with registered fumigants before use and re-use. Shipment should be covered using airtight containers or tarpaulins, temperature fluctuations that could lead to condensation should be avoided, this could lead to fungal growth and mycotoxin formation.

\subsection{Biological control measures}

The potential for using microorganisms to detoxify mycotoxins has been reported by Murphy, et.al. (2006) to be promising. One of the management strategies that had been developed is biological control using the competitive exclusion mechanism, which has been successfully implemented In the US, biological control has been used to reduce aflatoxin contamination in various crops such as cotton, maize and groundnut. The Internattional institute for agricultural research IITA,has pioneered this technique in Nigeria, by the development of its product called Aflasafe. Aflasafe has proven successful and is being tried on a number of crops. (Bandyopadhyay and Cardwell, 2003). 


\subsection{Physical methods of mycotoxin removal}

Once a contaminated product has reached a processing facility, clean-up and segregation are the first control options. In some cases, these are the best methods of reducing mycotoxin presence in final products. For example, when peanuts are processed, a significant amount of aflatoxins can be removed by electronic sorting and hand-picking ; separation of moulddamaged maize and/or screening can significantly reduce fumonisin and aflatoxin concentrations. In addition, the removal of rot from apples significantly reduces the patulin content in the final product. Although some contamination may persist, physical removal represents a good alternative for industry (Lopez-Garcia 1999). It is however important for grain handlers to use masks to avoid inhalation and ingestion of spores (as mould spores and mycotoxins are often concentrated in the fines and dust of grains). In general good agricultural practice entails a holistic approach and involves risk assessments throughout the process. These risk assessments can be performed at three points during the season: at the start of the season to assess the agronomic risk, at ear emergence to assess the need for spraying with insecticide and at harvest to assess the overall risk (Mohammed , 2006).

\subsection{Hazard Analysis Critical Control Point (HACCP)}

HACCP is a food management system designed to prevent safety problems, including food poisoning. It was adopted by the food industry because of its success. The HACCP approach involves conducting a detailed analysis of every step in a food processing operation by following seven clearly defined principles known as the HACCP plan (Adred et al. 2004). HACCP plan is an important aspect of an overall management approach in the control of mycotoxins, and these should include strategies for prevention, control, and quality from farm-to-fork. In the food industry, postharvest control of mycotoxins has been addressed via HACCP plans, which include the use of approved supplier schemes. Implementation at pre harvest stages of the food system through good agricultural practice (GAP) and post harvest stage through good manufacturing practice (GMP) is complementary to HACCP. Following an HACCP plan in mycotoxin control provides a critical front line defense to prevent introduction of contaminants into the food and feed supplies. Preharvest HACCP programs have been documented for controlling aflatoxin in corn and coconuts in Southeast Asia, peanuts and peanut products in Africa, nuts in West Africa, and patulin in apple juice and pistachio nuts in South America (FAO/IAEA2001). A number of HACCP schemes for wheat-based commodities were outlined by Aldred et al. (2004) from preharvest to atmospheric control in storage and Lopez-Garcia et al. (1999) provided guidance for development of an integrated mycotoxin management program.

\subsection{Mycotoxin regulations}

The framework legislation of a country is to ensure that any food containing a contaminant in an amount that is unacceptable from a public health point of view, particularly, at a toxicological level, cannot be marketed in that country. Contaminant levels are required to be kept as low as can reasonably be achieved by good practice. In most countries, 
regulations are established to control the contaminants in foodstuffs to protect human health; these regulations may include specific maximum limits for several contaminants for different foods and a reference to the sampling methods and performance criteria of analysis to be used (Mariko Kubo 2012). In 2003 the FAO carried out a worldwide international inquiry of mycotoxin regulations, which indicated that 100 countries had developed specific limits for mycotoxins in foodstuffs and feedstuffs. 99 countries had mycotoxin regulations for food and/or feed, an increase of approximately 30 percent compared to 1995. In 1995, 23 percent of the world's inhabitants were living in a region where no known mycotoxin regulations were in force. Countries with mycotoxin regulations in 2003 have at least regulatory limits for aflatoxin B1 or total aflatoxins (B1, B2, G1 and G2) in foods and/or feeds, specific regulations exist as well for aflatoxin M1; the trichothecenes, deoxynivalenol, diacetoxyscirpenol, T-2 toxin and HT-2 toxins; the fumonisins B1, B2, and B3; agaric acid; the ergot alkaloids; ochratoxin A; patulin; the phomopsins; sterigmatocystin and ochratoxins. The number of countries regulating mycotoxins has significantly increased over the years and more mycotoxins were now being regulated in more products

The inquiry also indicated that, there were 15 nations with known regulation in Africa (i.e. $59 \%$ of the inhabitants of Africa). For most African countries, specific mycotoxin regulations probably do not exist. However this does not mean that the problem of mycotoxin is ignored. Several of these countries recognize that they have problems due to

mycotoxins and that regulations should be developed. Aflatoxin has the highest mycotoxin regulations in Africa and Morocco had the most detailed mycotoxin regulations. Nigeria has adopted the European commission mycotoxin regulations which it uses primarily for export commodities, although these are applied in-country whenever there is a need to take a critical look especially on industrially processed food commodities. Consultations are currently on-going among regulatory agencies and other mycotoxin stakeholders in the country on the desirability or otherwise of retaining this status quo or creating a separate standard or set of standards.

\section{Challenges of mycotoxin research in Nigeria}

Although mycotoxin research started early in Nigeria in relation to the notoriety and worldwide realisation of the significance of aflatoxins, the world's leading mycotoxin, there remain a number of challenges that hinder mycotoxin research in Nigeria. These challenges include limited availability of equipment and kits, economic issues, adequate skills and experience, inadequate government support and inter-agency communications and inadequate funds directed at mycotoxin research among others.

Aflatoxin research in Nigeria began in 1961 after a team from the Tropical Products Research Institute in the United Kingdom visited Nigeria to alert the nation and those concerned with the export and production of groundnuts to the threat posed by aflatoxins. The government of the then Northern Nigeria set up a committee of government Departments and two Nigerian Research Institutes, the Institute of Agricultural Research 
(IAR) Samaru Zaria, and the Nigerian Stored Products Research (NSPRI) Ibadan (Blount 1961; Manzo and Misari 1989). Research at NSPRI relied mainly on thin layer chromatography (TLC), which which has been the classical method for mycotoxin analysis in Nigeria for several years and up till today (Arawora, 2010). Early work at IAR from 1961 - 1966 by MacDonald and colleagues relied on methods used in the laboratories of Tropical Products Research Institute London (McDonald Harness and Stone bridge 1965, Harkness et al, 1967). The Department of Crop Protection, IAR, established a mycotoxin research laboratory, in 1980 which has not been functional until recently when a team of researchers within the Department revived mycotoxin work there.

While TLC is a relatively easy technique for analysis, the challenge since the 1980's was the lack of mycotoxin standards which were expensive and not produced in the country. Most University researchers could not afford to carry out studies because standards had to be imported, and the universities barely had enough money to support expensive researches, due to the economic downturn caused by the introduction of the Structural Adjustment Programme (SAP) in 1986. Foreign companies were also sceptical of selling the standards because of the fear of its use for biological warfare. SAP thus resulted into poor funding of National Research Institutes and universities although a few scientists continued to carry out research on mycotoxins. The international institute for tropical agriculture (IITA) remained one of the institutions that continued to carry out mycotoxin research and held workshops to sensitize scientists (Bankole and Adebanjo 2003)

Currently, there is an upsurge of interests in mycotoxin research in Nigeria. New methods of analysis are already in use. These include High performance liquid chromatography (HPLC), Enzyme linked imunosorbent assay (ELIZA)) and more recently liquid chromatography with tandem mass spectroscopy (LC-MS/MS) which provides better quantification, larger and faster sample analysis and multitoxin analysis respectively (Wilson, 1989, Krska et al, 2008). Although some organisations and individuals can now afford to buy mycotoxin kits, the new challenge however is that they require the use of high-tech equipment. Most universities and research institutes in Nigeria do not use HPLC. There is no facility for LC-MS/MS analysis in the country at the moment, yet scientists have to ensure their researches are up to date. Researchers have to spend a large chunk of their money for analysis from the few organizations that have equipments, pay representatives of manufacturers of kits at a high cost or send their samples overseas for analysis.

Cooperation between organisations that have to do with mycotoxin research is also below expection. The universities and research institutes on one hand and Government organisations that regulate or formulate policies on mycotoxins need to have a more effective and formal relationship. The Mycotoxicology Society of Nigeria has been trying to fill this lacuna in the past seven years of its existence. The mandates of some of the government agencies such as the National Agency for Food and Drug Administration and Control (NAFDAC) and the standards Organisation of Nigeria (SON) do not allow them to do research. Some other agencies concerned with mycotoxin work are not in contact with the national research network. These agencies need to facilitate research for which they are 
beneficiary agencies. In Nigeria there is currently no Government agency or Department that specifically provides funds for or promotes mycotoxin research.

Skills for mycotoxin research are currently inadequate. The Mycotoxicological Society of Nigeria is currently the only organization that provides the cheapest training in Mycotoxin analysis through its annual conferences and workshops in collaboration with Mycotoxin kits manufacturers from around the world. The National agency for food and drug control (NAFDAC) and the International institute for tropical Agriculture (IITA) are the other organisations that provide training under certain arrangements. Foreign training such as (MYCORED) has so far come only through the provision of limited opportunities.

For Mycotoxin research to thrive, more capacity building through training, concerted effort of stakeholder organisations and Government to deliberately articulate and work out a mycotoxin research program in view of the importance of mycotoxins to human life and economy, is inevitable. This will immediately remove the constraints of funds and equipment which individual organisations and researchers are confronted with.

\section{Conclusion}

In many developing countries, the combination of insufficient drying and humid atmospheric conditions encourage mould growth and proliferation which results in unacceptable levels of mycotoxins especially aflatoxins in harvested maize, groundnuts, tree nuts and other agricultural produce.

Several studies in Nigeria have reported toxin levels far above the limits allowed by International regulatory agencies in food and agricultural products. In addition, fatal outbreaks of toxicities resulting particularly from aflatoxins have been widely reported in Nigeria. There is an urgent need to address the food safety and International trade issues associated with mycotoxin contamination in Nigerian consumer goods and agricultural products because there is a huge problem of mycotoxin contamination in developing countries like Nigeria where there exist a dearth of organized scientific information and data on the magnitude of the problem.

Furthermore, no single Governmental or private organization has the resources in personnel, expertise, money or time to mitigate against mycotoxin contamination. Therefore collaboration in projects involving multidisciplinary teams is needed for effective research, documentation, monitoring, evalution and control of mycotoxins in Nigeria.

Some of the suggested solutions to mycotoxin menace in Nigeria include:

1. Collection of a database of predominant fungi and mycotoxins in Nigeria

2. Construction of a Mycotoxin Occurrence Map to know the areas prone to Mycotoxin contamination

3. Establishment of a permanent culture collection centre

4. Good Agricultural practises (early planting and use of recommended crop production practices, irrigation to reduce drought stress, early harvesting, prevention of kernel 
damage during harvesting), Storage (adequate drying and proper storage below $13 \%$ moisture, and keeping storage facilities clean and dry) and Good Manufacturing Practices

\section{Author details}

Olusegun Atanda *

Mycotoxicology Society of Nigeria . Department of Biological Sciences. McPherson University, Km 96, Lagos- Ibadan Expressway, Seriki-Sotayo, Abeokuta, Ogun State, Nigeria

Hussaini Anthony Makun

Mycotoxicology Society of Nigeria, Department of Biochemistry, Federal University of Technology Minna, Niger State, Nigeria

Isaac M. Ogara

Mycotoxicology society of Nigeria. Department of Agronomy, Faculty of Agriculture, Nasarawa state university, Lafia campus, Lafia, Nigeria

Mojisola Edema

Mycotoxicology society of Nigeria. Department of Food Science \& Technology. Federal University of Technology, Akure, Nigeria

Kingsley O. Idahor

Mycotoxicology society of Nigeria. Department of Animal Science, Nasarawa State University, Keffi, Shabu-Lafia Campus, Lafia, Nigeria

Margaret E. Eshiett

Mycotoxicology society of Nigeria. Standards Organisation of Nigeria. Lagos, Nigeria

Bosede F. Oluwabamiwo

Mycotoxicology Society of Nigeria. National Agency for Food and Drug Administration and Control, Central Laboratory, Oshodi, Lagos, Nigeria

\section{References}

Abbas H. K., Cartwright R. D., Shier W. T., Abouzied M. M., Bird, C. B., Rice, L. G., Ross, P. F., Sciumbato, G .L. and Meredith, F.I. (1998). Natural Occurrence of fumonisins in rice with Fusarium sheat rot disease. Plant Disease, 82:22-25.

Abbas H. K., Gelderblom, W. C. A., Cawood, M.E. and Shier W.T. 1993. Biological activities of fumonisins, mycotoxins from Fusarium moniliforme in Jimsonweed (Datura stramonium L.) and mammalian cell cultures. Toxicon, 31:345-353.

Abbas H. K., Shier W. T. (2010). Mycotoxin contamination of agricultural products in the Southern United States and approaches to reducing it from pre-harvest to final food products.In: Appell M, Kendra D, Trucksess MW, eds. Mycotoxin Prevention and

${ }^{*}$ Corresponding Author 
Control in Agriculture. American Chemical Society Symposium Series. Oxford: Oxford University Press.

Abbas H. K., Shier, W. T., Cartwright, R. D. (2007). Effect of temperature, rainfall and planting date on aflatoxin and fumonisin contamination in commercial $\mathrm{Bt}$ and non-Bt corn hybrids inArkansas. Phytoprotection, 88:41-50.

Abalaka J. A. and Elegbede, J. A (1982). Aflatoxin distribution and total microbial count in an edible oil extracting plant 1: preliminary observations. Ed. Chem. Toxic 2:43-46

Abbas H. K., Williams W. P., Windham G. L., Pringle III H. C., Xie. W, Shier, W. T. (2002). Aflatoxin and fumonisin contamination of commercial corn (Zea mays) hybrids in Mississippi. Journal of Agriculture, Food and Chemistry, 50:5246-5254.

Adebajo L.O, Idowu A. A, Adesanya O. O. ( 1994). Mycoflora, and mycotoxins production in Nigerian corn and corn- based snacks. Mycopathologia; 126(3):183-92.

Adebajo, L. O. and Popoola, O. J. (2003) Mycoflora and mycotoxins in kolanuts during storage. African Journal of Biotechnology 2 (10):365-368

Adegoke G. O., Allamu A. E., Akingbala J. O. and Akanni A. O. (1996). Influence of sundrying on the chemical composition, aflatoxin content and fungal counts of two pepper varieties--Capsicum annum and Capsicum frutescens. Plant Foods in Human Nutrition. 49(2):113-7

Adejumo, O., Atanda, O. O, Raiola, A., Bandyopadhyay, R., Somorin, Y., Ritieni, A. (2012). Correlation between Aflatoxin $\mathrm{M}_{1}$ Content of Breast Milk, Dietary Exposure to Aflatoxin $\mathrm{B}_{1}$ and Socioeconomic Status of Lactating Mothers in Ogun State, Nigeria. Submitted to Food and Chemical Toxicology

Akande, K. E., Abubakar M. M., Adegbola T. A., Bogoro S. E. (2006) Nutritional and health implications of mycotoxins in animal feeds: a review. Pakistan Journal of Nutrition 5 (5): 398-403.

Akano, D. A. and Atanda, O. O. (1990). The present Level of Aflatoxin in Nigeria Groundnut Cake ("Kulikuli"). Letters in Applied Microbiology. 10: 187 - 18

Alberts J. F., Engelbrecht, Y., Steyn P. S., Holzapfel W. H., van Zyl W. H. ( 2006 ) . Biological degradation of aflatoxin $\mathrm{B}_{1}$ by Rhodococcus erythropolis cultures. International Journal of Food Microbiology 109, (1-2): 121-126.

Aldred D., N. Magan, and M. Olsen (2004) The use of HACCP in the control of mycotoxins: the case of cereals. In Magan N. and Olsen M(Ed.) Mycotoxins in food: Detection and Control. Woodhead Publishing in Food Science and Technology. pp 139-173.

Amujoyegbe B. M. (2012). Farming systems analysis of two agro-ecological zones of SouthWestern Nigeria. Resjournals .com/ARJ pp1-12

AFRO Food Safety Newsletter Issue No 2. July 2006 World Health Organization Food Safety (FOS)African Society of Toxicological Sciences (ASTS) March 1920, 2009, Baltimore, AST S Satellite Meeting

Aregheore, E. M. ( 2005) Nigeria Country profile. www.fao.org/ag/AGP/AGPC/doc/Couprofile

Arowora, K. A. and J. N. Ikeorah (2010) Five decades of Aflatoxin research in NSPRI. Fifth annual conference of the Nigeria Mycotoxin awareness research and study network, Nigeria stored products research Institute, Ilorin. 26-28 $8^{\text {th }}$ September 2010. 
Aroyeun, S. O. and Adegoke, G. O. (2007) Reduction of Ochratoxin A (OTA) in spiked cocoa powder and beverage using aqueous extracts and essential oils of Aframomum danielli. African Journal of Biotechnology. 6(5) :612-616.

Atanda, O. O., Oguntubo, A., Adejumo, A., Ikeorah, H. and Akpan, I. (2007). Aflatoxin M1 contamination of Milk and Ice-cream in Abeokuta and Odeda Local Governments of Ogun State, Nigeria. Chemosphere. 68: 1455-1458.

Atehnkeng, J., Ojiambo, P.S., Ikotun, T., Sikora, R.A., Cotty, P.J., Bandyopadhyay, R., 2008. Evaluation of atoxigenic isolates of Aspergillus flavus as potential biocontrol agents for aflatoxin in maize. Food Additives and contaminants: Part A 25, 1264-1271.

Avantaggio, G., Quaran, F., Desidero, E. and Visconti, A. (2002). Fumonisin contamination of maize hybrid visibly damaged by Sesame. J. Sci. Food Agric. 83:13-18.

Ayodele B. C. and Edema M. O. (2010). Evaluation of the critical control points in the production of dried yam chips for elubo. Nigerian Food Journal.

Bacon C. W., Porter J. K. and Norred, W. P. (1995). Toxic interaction of fumonisin B, and fusaric acid measured by injection into fertile chicken egg. Mycopathologia, 129:29-35.

Bankole S. A. and Adebanjo, A. (2003) Mycotoxins in food in West Africa: current situation and possibilities of controlling it. African Journal of Biotechnology. 2(9): 254-263.

Bankole S. A. and Mabekoje, O. O. (2004). Occurrence of aflatoxins and fumonisins in preharvest maize from South-Western Nigeria. Food Addict Contam. (3):251-5.

Bankole S. A, Ogunsanwo, B. M. and Mabekoje O .O. (2004). Natural occurrence of moulds and aflatoxin $B_{1}$ in melon seeds from markets in Nigeria. Food and Chemical Toxicology, 42(8):1309-14.

Bankole S. A.,Ogunsanwo, B. M., Eseigbe, D.A., (2005). Aflatoxins in Nigerian dry-roasted groundnuts. Food Chemistry. 89 (4), 503-506

Bassir, O. and Adekunle, A. (1969). Comparative toxicities of Aflatoxin B1 and palmotoxins Bo and Go West African Journal of Biological and Applied Chemistry, 12(1):7-19.

Bassir, O. (1969). Toxic substance in foodstuffs. West African Journal of Biological and Applied Chemistry, 12:4-7

Becker B., Bresch H., Schillinger U. and Thiel P.G. 1997. The effect of fumonisin B 1 on the growth of bacteria. World Journal of Microbiology and Biotechnology, 13:539-543.

Bennett, J.W., Klich, M., (2003). Mycotoxins. Clinical Microbiology Reviews, 16, 497-516.

Bhat, R.V. and S. Vasanthi, (2003). Food Safety in Food Security and Food Trade. Mycotoxin Food Safety Risk in Developing Countries. International Food Policy Research Institute.

Bhat, R., Rai, R. V., \& Karim, A. A. (2010). Mycotoxins in Food and Feed: Present Status and Future Concerns. Comprehensive Reviews in Food Science and Food Safety, 9: 57-81

Blount, W. P. (1961). Turkey ' $X$ ' Disease. J. Brit. Turkey federation. 9: 52-61.

Bothast R. J., Bennett, G. A., Vancauwenberge, J .E. and Richard J. L. (1992). Fate of fumonisin B in naturally contaminated corn during ethanol fermentation. Applied Environmental Microbiology, 58:233-236.

Boutrif E. (1995). FAO programmes for prevention, regulation, and control of mycotoxins in food. Natural Toxins 3 (4), 322-326.

Brown, D. W., McCoy C. P. and Rottinghaus G. E. 1994. Experimental feeding of Fusarium moniliforme culture material containing furnonisin B, to channel catfish (Ictalurus punctatus). Journal of Veterinary Diagnostic Investigation, 6:123-124 
Bullerman, L. B. (2007). Mycotoxins from Field to Table. International journal of food Microbiology. 119 (1-2) 131-139

Center for Disease Control and Prevention. (2004). Outbreak of aflatoxin poisoning-eastern and central provinces, Kenya, January-July 2004. 53(34):790-793.

Codex Alimentarius Commission (2003). "Proposed Draft Code of Practice for the Prevention (Reduction) of Mycotoxin Contamination in Cereals, Including Annexes on Ochratoxin A, Zearalenone, Fumonisins and Tricothecenes." Codex Committee on Food Additives and Contaminants, Thirty-fourth Session. Codex Alimentarius Commission /RCP 51-2003

Cole R. J., Doner JW, Holbrook CC. (1995). Advances in mycotoxin elimination and resistance. In: Pattee HE, Stalker H.T, (eds). Advances in Peanut Science. Stillwater, OK: American Peanut Research and Education Society, pp. 456-474.

Dada. J. D. (1978). Studies of fungi causing grain mould of sorghum varieties in northern Nigeria with special emphasis on species capable of producing mycotoxins. M.Sc. thesis, Ahmadu Bello University, Zaria.

Darling, S. J. (1963).Research on aflatoxin in groundnuts in Nigeria. 13. Institute of Agric. Research, Ahmadu Bello University, Samaru, Zaria.

Diener, U. L., Cole, R. J., Sanders, T. H., Payne, G. A., Lee, L. S., Klich, M. A., (1987). Epidemiology of Aflatoxin, in formation by Aspergillus flavus. Annual Review of Phytopathology 25, 240-270.

D'Mello, J. P. F., MacDonald, A. M. C., 1997. Mycotoxins. Animal Feed Science and Technology, 69, 155-166.

Doehlert, D. C., Knutson C.A. and Vesonder R. F. (1994). Phytotoxic effects of fumonisin B, on maize seedling growth.Mycopathologia, 127:117-121.

Dohlman E. (2003). Mycotoxin Hazards and Regulations: Impacts on Food and Animal Feed Crop Trade. In International Trade and Food Safety: Economic Theory and Case Studies. pp 96.

Edema M. O. and Adebanjo, F. (2000) Distribution of Myco-flora of some sun-dried vegetables in South-western Nigeria. Nigerian Journal of Microbiology 14 (2): 119-122.

Elegbede, J. A. (1978). Fungal and Mycotoxin contamination of Sorghum during storage. M.Sc. thesis. Department of Biochemistry, Ahmadu Bello University, Zaria.

Ewuola E. O., Ogunlade J.T., Gbore F. A., Salako A. O., Idahor K. O. and Egbunike G. N. (2003). Performance Evaluation and Organ Histology of Rabbits fed Fusarium verticillioides culture Material. Trop. Anim. Prod. Invest., 6:111-119.

Ezekiel, C. N., Sulyok, M., Warth, B and Krska, R (2012a). Multi-microbial metabolites in fonio millet (acha) and sesame seeds in Plateau State, Nigeria. European Food Research Technology, 235: 285-2

Ezekiel, C. N., Bandyopadhyay, R., Sulyok, M., Warth, B and Krska, R (2012b). Fungal and bacterial metabolites in commercial poultry feed from Nigeria. Food Additives and Contaminants: Part A. 29 (8): 1288-1299

Ezekiel, C. N., M. Sulyok, D. A. Babalola, B. Warth, V. C. Ezekiel, Krska, R. (2012c). Incidence and consumer awareness of toxigenic Aspergillus section Flavi and aflatoxin B1 in peanut cake from Nigeria. Accepted manuscript Food Control. 
Ezekiel C. N., M. sulyok, B. warth, A. C. odebode and R. krska (2012). Natural occurrence of Mycotoxins in peanut cake from nigeria. Food control. 27:338-342.

Fandohan, P., Gnonlonfin, B., Hell, K., Marasas, W.F.O., Wingfield, M.J.,( 2005). Natural occurrence of Fusarium and subsequent fumonisin contamination in preharvest and stored maize in Benin, West Africa. International Journal of Food Microbiology, 99, 173183.

Gbodi T.A. Nwude, N, Aliu, Y.O, and Ikediobi, C.O. (1984). Mycotoxins and Mycotoxicoses, the Nigerian situation to Date. National Conference on Disease of Ruminant. $3^{\text {rd }}$ and $6^{\text {th }}$ October, 1984. National Veterinary Research Institute, Vom. pp. 108-115.

Gbodi, T.A. (1986). Studies of mycoflora and mycotoxins in Acha, maize and cotton seed in plateau state,Nigeria. Ph. D. thesis. Department of Physiology and Pharmacology, Faculty of Veterinary Medicine, A.B.U, Zaria, pp 213.

Goel S., Lenz S. D., Lumlertdacha, S., Lovell R. T., Shelby R. A., Li, M., Riley, R. T. and Kemppainen B., W. (1994). Sphingolipid levels in catfish consuming Fusaruim moniliforme corn culture material containing fumonisins. Aquatic Toxicology, 30:285294.

Gross S. M., Reddy, R. V., Rottinghaus, G.E., Johnson G.and Reddy C.S. (1994). Developmental effects of fumonisin $\mathrm{B}_{1}$ - containing Fusarium moniliforme culture extract in CDI mice. Mycopatholgia, 128:11-118.

Gong Y. Y., K Cardwell, A. Hounsa, S. Egal, P. C. Turner, A. J. Hall, C. P., Wild (2002). Dietary aflatoxin exposure and impaired growth in young children from Benin and Togo: cross sectional study. British Medical Journal. 325:20.

Halliday, D. (1965) the aflatoxin content of Nigeriangroundnuts and cake. Nigerian Stored Products Research Institute, Technical Report, Lagos.

Halliday, D. (1966). Further studies of the aflatoxin content of Nigerian groundnuts and groundnut products. Nigerian Stored Products Research Institute, Technical Report, Lagos.

Halliday, D. \& Kazaure, I. (1967). The aflatoxin content of Nigerian Groundnut cake. Nigerian Stored Products Research Institute Technical report No. 8.

Harkness C., D. McDonald, W.C. Stonebridge, J. A'Brook and H. S. Darling (1966) The problem of Aflatoxin in Groundnuts (Peanuts) and other food crops of tropical Africa. Food technology. 20(9): 152-163.

Hussein, H.S., Brasel, J.M.,( 2001). Toxicity, metabolism, and impact of mycotoxins on humans and animals. Toxicology 167, 101-134.

IARC. (1993). Toxins derived from Fusarium moniliforme: Fumonisms $B_{1}$ and $B_{2}$ and fusarin C. In: Some naturally occurring substances: Food items and constituents, heterocyclic aromatic amines and mycotoxins. 56:445-466.

Idahor K. O., Adgidzi, E .A. and Usman, E. A. ( 2010). Awareness of the association of mycotoxins with food and feedstuffs in Nasarawa State. $5^{\text {th }}$ Ann. Conf. Nigeria Mycotoxin Awareness and Study Network, Ilorin. Kwara State. 25pp.

Idahor K. O. (2010). Effects of Fumonisin B1 on Living Organisms. Production Agriculture and Technology Journal, 6 (1):49-65. 
Idahor K. O., Gbore F. A., Salako A. O., Weuola E. O, Ogunlade J. T. and Egbunike G. N. (2008a). Physiologic response of rabbits to fumonisin $\mathrm{B}_{1}$ dosed orally with maize-based diets. Production Agriculture and Technology Journal, 4(1):91-98.

Idahor K. O., Ewuola E. O., Gbore F. A., Ogunlade J. T., Salako O. A. and Egbunike G. N. (2008b). Reproductive performance of rabbits fed maize-based diets containing FB 1 strain of Fusarium verticillioides (Sacc.). Production Agriculture and Technology Journal, 4 (2): 85-92.

Ifeji, E. (2012). Fungi and some mycotoxins found in groundnuts (Arachis hypogea)from Niger State, Nigeria. M. Tech Dissertation. Department of Biochemistry. Federal University of Technology, Minna, Nigeria.

Ikwuegbu, O. A. (1984). Two decades of Aflatoxin Research in Vom. National Conference on Diseases of Ruminants $3^{\text {rd }}-6^{\text {th }}$ of October, 1984. National Veterinary Research Institute, Vom. pp100-106.

Jantratail, W., Lovell, R. T. (1990). Subchronic toxicity of dietary aflatoxin B 1 to channel catfish. Journal of Aquatic Animal Health, 2: 248-254.

JECFA (2001). WHO Food additives series 47: Safety Evaluation of certain mycotoxins in foods. Joint FAO/WHO expert committee on food additives. 701pp.

Kaaya, A. N., Kyamuhangire, W., Kyamanywa, S. (2006). Factors affecting aflatoxin contamination of harvested maize in the three agro-ecological zones of Uganda. Journal of Applied Sciences 6, 2401-2407.

Kaneshiro T., Resonder R. F. and Peterson R. E. (1992). Fumonisin-stimulated Nacetyldihydro-sphingosine, N- acelyl- phytosphingosine and phytosphingosine products of Pichia ciferri (Hansenula), NRRL Y-1031. Current Microbiology, 24:319-324.

Krska, R., P. Schubert-Ullrich, M. Sulyok, S. MacDonald and C. Crews (2008) Mycotoxin analysis: an update. Food additives and contaminants. 25(2): 152-163.

Kubo, M. (2012) Mycotoxins Legislation Worldwide. European Mycotoxin Nettwork/ Leatherhead Food Research.www.Mycotoxin.com

Kumar V., M. S. Basu, T. P., Rajendran (2008). Mycotoxin research and mycoflora in some commercially important agricultural commodities. Crop Protection. 27 (6), 891-905.

Lacey, T. (1986). Factors affecting mycotoxin production. In: Mycotoxins and phycotoxins edited by Steyn, P.S. and Vlegaar, R. $6^{\text {th }}$ international IUPAC symposium on mycotoxins and phycotoxins, Pretoria, South Africa.

Lamprecht S. C., Marasas W. F. O., Alberts J. F., Cawood M. E., Gelderbcom W. C. A., Shephard G. S., Thiet P. G and Calitz F. J. (1994). Phytotoxicity to fumonisins and TAtoxin to corn and tomate. Phytopathology, 84:383-391.

Lanyasunya, T. P., Wamae, L. W., Musa, H. H., Olowofeso, O., Lokwaleput, I. K., (2005). The risk of mycotoxins contamination of dairy feed and milk on smallholder dairy farms in Kenya. Pakistan Journal of Nutrition, 4, 162-169.

Leslie, J. Bandyopadhyay R. and Visconti A., (2008). Mycotoxins: Detection Methods, Management, Public Health and Agricultural Trade CABI. 464pp.

Liu, Y. and Wu, F. (2010). Global Burden of Aflatoxin-induced Hepatocellular Carcinoma: A Risk Assessment. Environmenral Health Perspectives. 118(6): 818-824

Logrieco A., Doko M.B., Moretti A., Frisullo S. and Visconti A. (1998). Occurrence of fumonisin $B_{1}$ and $B_{2}$ in Fusarium proliferation infected asparagus plants. Journal of Agricultural and Food and Chemistry, 46:5201-5204. 
Lopez-Garcia, R., Park, D., (1998). Effectiveness of post-harvest procedures in management of mycotoxin hazards. In: Bhatnagar, D., Sinha, S. (Eds.), Mycotoxins in Agriculture and Food Safety. Marcel Dekker, New York, USA, pp. 407-433.

Lopez-Garcia R., Park D. L. and Phillips T. D. (1999). Integrated mycotoxin management systems. Food Nutrition and Agriculture/ANA 23, (4) 38-48

Lowe D. P. and Arendt, E. K. (2004) The Use and Effects of Lactic Acid Bacteria in Malting and Brewing with Their Relationships to Antifungal Activity, Mycotoxins and Gushing: A Review. Journal of Institute of Brewing, 110(3), 163-180.

Lubulwa, A. S. G. and Davis, J. S. (1994). Estimating the social costs of the impacts of fungi and aflatoxins. In Highley, E., Wright, E.J., Banks, H.J. and Champ, B.R. (eds.) Stored Products protection, Proceedings of the $6^{\text {th }}$ International Working Conference in Storedproduct Protection, 17-23 April 1994, Canberra, Australia. CAB International, Wallingford, UK.

McDonald, D. C. Harkness and W. C. Stonebridge (1965). Growth of Aspergillus flavus and production of Aflatoxin in groundnuts. Part VI. Samaru research bulletin. Institute of Agricultural research, Ahmadu Bello Universtity, zaria.

Mclean, M. and P., Berjak, (1987). Maize grains and their associated mycoflora: A microecological consideration. Seed Science \& Technology, 15: 813-850.

Makaula N. A., Marasas W. F.O., Venter F. S., Badenhorst C.J. Bradshaw D. and Swanevelder, S. (1996). Oesophageal and other cancer patterns in four selected districts of Transkei, Southern Africa: 1985-1990. African Journal of Health Science,3:11-15.

Makun, H. A., Timothy, A. Gbodi, O, H., Akanya, A., Ezekiel, Salako, E.A.and Godwin, H. Ogbadu. (2007). Fungi and some mycotoxins contaminating rice (Oryza sativa) in Niger State, Nigeria. African Journal of Biotechnology 6 (2): 99 - 108.

Makun, H. A. , Gbodi T. A., Akanya, H. O. , Sakalo, A. E. and Ogbadu, G. H. (2009a). Health implications of toxigenic fungi found in two Nigerian staples: guinea corn and rice. African Journal of Food Science, 3: 250-256.

Makun, H. A., Gbodi, T. A., Akanya, H. O., Salako, E. A. and Ogbadu, G. H. (2009b). Fungi and some mycotoxins found in mouldy Sorghum in Niger State, Nigeria. World Journal of Agricultural Sciences. 5 (1): 05 - 17.

Makun, H. A. , Anjorin S. T., Moronfoye, B., Adejo, F. O., Afolabi, O. A., Fagbayibo, G., Balogun, B.O. and Surajudeen, A. A. (2010). Fungal and aflatoxin contaminations of some human food commodities in Nigeria. African Journal of Food Sciences. 4 (4): 127 - 135.

Makun H. A., M. F. Dutton, P. B. Njobeh, M. Mwanza and Kabiru, A. Y. (2011). Natural multi- mycotoxin occurrence in rice from Niger State, Nigeria. DOI: 10.1007/s12550-0100080-5. Mycotoxin Research. 27 (2): 97-104

Makun H. A. , Mailafiya, C. S., Saidi, A. A., Onwuike, B. C. and Onwubiko, M. U. (2012). A preliminary survey of aflatoxin in fresh and dried vegetables in Minna, Nigeria. African Journal of Food Science and Technology 3(10) pp. xxx-xxx,

Manorama, S., Singh, R., (1995). Mycotoxins in milk and milk products. J. Dairying, Foods Home Sci. 14, 101-107.

Mantle, P. G. (1968) Studies on Sphacelia sorghi McRae, an ergot of Sorghum vulgare Pers. Annals of applied Biology. 62(3) 443-449 
Manzo, S. K. and S. M. Misari (1989) Status and management of Aflatoxin in Groundnuts in Nigeria. In McDonald, D. And V.K. Mehan (eds.). Aflatoxin contamination of Groundnuts: Proceedings of the international workshop, ICRISAT center, India.77-87.

Marasas, W. F. O., Van Rensburg S. J. and Mirocha C. J. (1979). Incidence of Fusarium species and the mycotoxins, deoxynivalenol and zearalenone, in corn produced in oesophageal cancer areas in Transkei, Journal of Agricultural and Food Chemistry, 27:1108-1112.

Meister, U. Symmank, H. and Dahlke, H. (1996). Investigation and Evaluation of the contamination of native and imported cereals with fumonisins.) $Z$ Lebensm Unters Forseh, 203: 528-533.

Meredith, F. I., Riley R. T., Bacon C. W., Williams D. E. and Carlson D. B (1998). Extraction, quantification and biological availability of fumonisin $\mathrm{B}_{1}$ incorporated into Oregon test diet and fed to rainbow trout. Journal of Food Protection, 61:1034-1038.

Mestres, C., Bassa, S., Fagbohun, E., Nago, M., Hell, K., Vernier, P., Champiat, D., Hounhouigan, J., and Cardwell, K.F. (2004). Yam chip food sub-sector: hazardous practices and presence of aflatoxins in Benin. Journal of Stored Products Research. 40, 575-585.

Milicevic, D., Juric, V., Stefanovic, S., Jovanovic, M., Jankovic, S. (2008). Survey of slaughtered pigs for occurrence of ochratoxin A and porcine nephropathy in Serbia. International Journal of Molecular Science, 9, 2169-2183.

Mohammed H. K. (2012). Fungi and some mycotoxins contaminating maize from Niger State, Nigeria. M. Tech Dissertation. Federal University of Technology, Minna. Nigeria.

Munkvold, G. P. (2003). Cultural and genetic approaches to managing mycotoxins in maize. Annual Review of Phytopathology, 41, 99-116.

Murphy P. A., Hendrich S., Landgren C. and Bryant C.M. (2006). Food Mycotoxins: an Update. A scientific status summary. Journal of Food science 7(5) R51-R65 doi:10.1111/j.1750-3841.2006.00052.x

Naresh Magan and David Aldred International Journal of Food Microbiology, Volume 119: $1-2$.

Negedu, A., Atawodi, S. E., Ameh, J. B., Umoh, V.J., and Tanko, H.Y. (2011). Economic and health perspectives of mycotoxins: a review. Continental Journal of Biomedical Sciences, 5 (1): 5 - 26

Obasi O. E, Ogbadu G. H. and Ukoha A. I. (1987). Aflatoxins in Burukutu (Millet Beer). TransTrTransactions of Royal Society of Tropical Medicine and Hygiene, 81: 879

Obidoa, O and Gugnani, H. C. (1990). Mycotoxins in Nigerian foods: causes, Consequences and remedial measures. In: Okoye, Z.S.C Mycotoxins contaminating foods and foodstuffs in Nigeria. First National Workshop on Mycotoxins. 29 $9^{\text {th }}$ November, 1990. University of Jos . pp. 95-114.

Ocholi R. A, Chima, J. C. , Chukwu, C. O. , Irokanulo, E. (1992). Mycotoxicosis associated with Penicillium purpurogenum in horses in Nigeria. Veterinary Record, 130(22):495.

Ogunlade, J. T., Gbore F. A., Ewuola E. O., Idahor K. O., Salako A. O. and Egbunike G. N. (2004). Biochemical and haematological response of rabbits fed diets containing micro doses of fumonisin. Tropical Journal of Animal Science, 7(1): 169-176.

Okeke, K. S., Abdullahi, I. O., Makun H. A. and Mailafiya, S. C. (2012). A preliminary survey of aflatoxin M1 in dairy cattle products in Bida, Niger State, Nigeria. African Journal of Food Science and Technology. 3(10) pp. xxx-xxx, 
Okonkwo, P. O. and Nwokolo, C. (1978). Aflatoxin B1: sample procedure to reduce levels in tropical foods. Nutrition Reports International, 17(3): 387-395.

Okoye, Z. S. C. and Ekpenyong, K. I. (1984). Aflatoxins B 1 in native millet beer brewed in Jos suburb. Transactions of Royal Society of Tropical Medicine and Hygiene, 78; 417-418.

Okoye, Z. S. C. (1986). Zearalenone in native cereal beer in Jos Metropolis of Journal of Food safety, 7;233-239.

Okoye, Z. S. C. (1992). An overview of Mycotoxins likely to contaminate Nigerian staple food stuff. First National Workshop on Mycotoxins. 29 $9^{\text {th }}$ November, 1990 . University of Jos. pp. 9-27.

Okoye Z. S. C. (1993) Fusarium mycotoxins nivalenol and 4-acetyl-nivalenol (fusarenon-X) in mouldy maize harvested from farms in Jos district, Nigeria. Food Addit ives and Contaminants;10(4):375-9.

Olorunmowaju, B. Y. (2012). Fungi and mycotoxins contaminating rice (Oryza sativa) from Kaduna State. M. Tech Dissertation. Department of Biochemistry. Federal University of Technology, Minna, Nigeria

Oluwafemi F. and Da-Silva F. A. (2009) Removal of aflatoxins by viable and heat-killed Lactobacillus species isolated from fermented maize. Journal of Applied Biosciences 16: $871-876$.

Oluwafemi, F., and Taiwo, V. O. (2003). Reversal of toxigenic effects of aflatoxin $B_{1}$ on cockerels by alcoholic extract of African nutmeg Monodora myristica. Journal of the Science of Food and Agriculture, 84: 333-340.

Ominski, K. H. (1994). Ecological aspects of growth and toxin production by storage fungi. In: Miller, J.D., Trenholm, H. S. (Eds.). Mycotoxin in grains: Compounds other than aflatoxin. Eagan press, USA. pp. 287-305.

Onilude A. A, Fagade, O. E, Bello, M. M, Fadahunsi, I. F. (2005). Inhibition of aflatoxinproducing aspergilli by lactic acid bacteria isolates from indigenously fermented cereal gruels. African Journal of Biotechnology, 4(12):1404-1408.

Onyemelukwe, G. C. and Ogbadu, G. H and Salifu, A. (1982). Aflatoxin B, G. and G2 in primary liver cell carcinoma. Toxicology Letters, 10:309-312.

Opadokun, J. S. (1992). Occurrence of Aflatoxin in Nigeria food crops. First National Workshop on Mycotoxins. 29 th November, 1990. University of Jos. pp. 50-60

Otsuki, T., Wilson, J. S. and Sewadeh, M. (2001a). A Case Study of Food Safety Standards and African Exports. World Bank Policy Research working paper N0. 2563, World Bank, Washington, D.C.

Otsuki, T., Wilson, J. S., and Sewadeh, M. (2001b) What price precaution? European harmonisation of aflatoxin regulations and African groundnut exports. European Review of Agricultural Economics, 28: 263-283.

Oyelami O. A., S. M. Maxwell, K. A. Adelusola, T. A. Aladekoma and A. O. Oyelese (1995) Aflatoxins in the autopsy brain tissue of children in Nigeria. Mycopathologia, 132 (1), 35-38.

Oyelami O. A., S. M. Maxwell K. A. Adelusola T. A. Aladekoma A. O. Oyelese (1998) Aflatoxins in autopsy kidney specimens from children in Nigeria. Journal of Toxicology and Environmental Health. 55 (5): 317-323. 
Oyejide A, Tewe O. O., Okosum S. E. (1987). Prevalence of aflatoxin B1 in commercial poultry rations in Nigeria. Beitr Trop Landwirtsch Veterinarmed.; 25(3):337-41

Payne, G. A. (1992). Aflatoxins in maize. Critical Rev Plant Sci.10:423-440.

Peers, F. G, (1965). Summary of the work done in Vom (Northern Nigeria) on Aflatoxin levels in groundnut flour. Agric nutr. Document R. 3/Add. P.A.G (WHO/FAO/UNICEF) Rome.

Rachaputi, N. R., Wright G. C., Kroschi, S. (2002). Management practices to minimise preharvest aflatoxin contamination in Australian groundnuts. Australian Journal of Experimental Agriculture, 42: 595-605.

Bandyopadhyay, R. , S., Kiewnick, J. Atehnkeng, Donier, M. (2005). Biological control of aflatoxin contamination in maize in Africa International Institute of Tropical Agriculture (IITA), Plant Health Management Program, Ibadan, Nigeria.

Rauber, R. H., Dilkin, P., Giacomini, L. Z., Araujo de Almeida, C. A., Mallmann, C. A. (2007). Performance of Turkey Poults fed different doses of aflatoxins in the diet. Poultry Science, 86: 1620-1624.

Ross P.F, Ledet A. E., Owens D. L., Rice L. G., Nelson H. A., Osweiler G. D. and Wilson T. M. (1993). Experimental equine leukoencephalomalacia, toxic hepatosis and encephalopathy caused by corn naturally contaminated with fumonisins. Journal of Veterinary Diagnostic Investment, 5:69-74. 998.

Rustemeyer, S. M., Lamberson, W. R., Ledoux, D. R., Rottinghaus, G. E., Shaw, D. P., Cockrum, R. R., Kessler, K. L., Austin, K. J., \& Cammack, K. M. (2010). Effects of dietary aflatoxin on the health and performance of growing barrows. Journal of Animal Science, Vol. 88, Nov, pp. 3624-3630,0021-8812.

Salifu, A., 1978. Mycotoxins in short season varieties of Sorghum in Northern Nigeria. Samaru Journal of Agriculture Research, 1: 83-87.

Shetty, P. H. and Bhat, R. V. (1997). Natural occurrence of fumonisin B 1 and its cooccurrence with aflatoxin $\mathrm{B}_{1}$ in Indian sorghum, maize and poultry feeds. Journal of Agriculture, Food and Chemistry, 45:2170-2173.

Shier, W. T., Abbas H. K., Abou-Karam, M., Badria, F. A, Resch, P.A. (2003). Fumonisins: Abiogenic conversions of an environmental tumor promoter and common food contaminant. Journal of Toxicology-Toxin Reviews, 22:591-616.

Shier, W. T., Abbas H.K , Badria, F. A. (1997). Structure-activity relationships of the corn fungal toxin fumonisin B1: implications for food safety. Journal of Natural Toxins, 6:225-242.

Shier, W. T., Tiefel, P.A. , Abbas, H. K. (1999). Current research on mycotoxins: fumonisins. In: Tu AT, Gaffield W, (eds). Natural and Selected Synthetic Toxins: Biological Implications. American Chemical Society Symposium Series. 745. Oxford, UK: Oxford University Press, pp. 54-66.

Smith, J. S. and Thakur, R. A. (1996). Occurrence and fate of fumonisins in beef. Advances in Experimental Medicine and Biology, 392:39-55.

Sun, T. S. C. and Stahr, H. M. (1993). Evaluation and application of a bioluminescent bacterial genotoxicity test. Journal of the Association of Official Analytical Chemists, 76: 893-898. 
Sydenham, E. W., Thiel, P.G., Marasas, W. F. O., Shephard G.S., Van Schalkwyk D.J. and Koch K. R. (1990). Natural Occurrence of some Fusarium mycotoxins in corn from low and high oesephageal cancer prevalence area of the Transkei, Southern AfricaJournal of Agricultural and Food Chemistry, 38:1900-1903.

Teniola, O. D., Addo, P. A., Brost, I. M., Farber, P., Jany, K .D., Albert, J. F., zanZyl, W. H., Steyn, P. S., Holzapfel, W. H., (2006). Degradation of aflatoxin B1 by cell-free extracts of Rhodococcus erythropolis and Mycobacterium fluoranthenivorans sp. nov. DSM 44556T. International Journal of Food Microbiology, 105:111-117.

Tijani, A. S. (2005). Survey of fungi, aflatoxins and zearalenone contamination of maize in Niger State. M.Sc thesis Department of Biochemistry, Federal University of Technology. Minna.

Trenk, H. L. and Hartman P. A. (1970). Effects of Moisture Content and Temperature on Aflatoxin Production in Corn. Applied Microbiology. 19 (5): 781-784.

Torres, M. R., Sanchis V. and Ramos A. J. (1998). Occurrence of fumonisins in Spanish beers analyzed by an enzyme- linked immunosorbent assay method. International Journal of Food Microbiology, 39:39-143.

Upadhaya, S. D., Park, M. A., \& Ha, J. K. (2010). Mycotoxins and Their Biotransformation in the Rumen: A Review. Asian-Australasian Journal of Animal Sciences, 23, 1250-126.

Uriah, N. and Ogbadu, L. (1982). Influence of woodsmoke on aflatoxin production by Aspergillus flavus. European Journal of Applied Microbiology and Biotechnology, 14:5153.

USNTP (1999). Toxicology and carcinogenesis studies of fumonisin $B_{1}$ in rats and mice. NTP Technical report. pp. 99-3955.

van Burik J. and Magee P.T. (2001). Aspects of fungal pathogenesis in humans. Annal Review of Microbiology, 55: 743-772.

Vesonder R. F., Labeda D. P. and Peterson R. E. (1992). Phytotoxic activity of selected watersoluble metabolites of Fusarium against Duckweed (Lemma minor L.). Mycopathlogia, 118: 185-189.

Voss, K. A., Chamberlam, W. J., Bacon, C. W., Herbert, R. A., Walters, D. B. and Norred, W .1. P. (1995). Subchronic feeding study of the mycotoxin fumonisin $B_{1}$ in mice and rats. Fundamentals of Apllied Toxicology, 24:102-110.

Wilson, D. M. (1987). Detection and determination of Aflatoxins in Maize. In zuber, M.s., E.B. Lillehojand B. Reinfro (eds.) Aflatoxins in Maize: A proceedings of the international workshop. CIMMYT, Mexico D.F. 100-109

Wilson, T. M., Ross P. F., Owens, D. L., Rile, L. G., Green, S. A., Jenkins, S. J. and Nelson, H. A. (1992). Experimental reproduction of ELEM-A study to determine the minimum toxic dose in ponies. Mycopathologia, 117:115-120.

Wu W-I, McDonough V. M., Nickels J. T., Ko S. J., Fischl A. S., Vales T. R., Merrill A. H. Jr. and Carman G. M.(1995). Regulation of lipid biosynthesis in Saccharomyces cerevisiae by fumonisin B1. Journal of Biological Chemistry, 270: 13171 - 13178.

Yan, L.-Y., Yan, L.-Y., Jiang, J.-H., Ma, Z.-H. (2008) . Biological control of aflatoxin contamination of crops. Journal of Zhejiang University - Science B 9, 787-792.

Zain, M. E. (2011). Impact of mycotoxins on humans and animals. Journal of Saudi Chemical Society. 15, 129-144. 